\title{
A STOCHASTIC PROGRAMMING APPROACH FOR SHELTER LOCATION AND EVACUATION PLANNING
}

\author{
VEDAT BAYRAM ${ }^{1}$ AND HANDE YAMAN Y,* $^{2, *}$
}

\begin{abstract}
Shelter location and traffic allocation decisions are critical for an efficient evacuation plan. In this study, we propose a scenario-based two-stage stochastic evacuation planning model that optimally locates shelter sites and that assigns evacuees to nearest shelters and to shortest paths within a tolerance degree to minimize the expected total evacuation time. Our model considers the uncertainty in the evacuation demand and the disruption in the road network and shelter sites. We present a case study for a potential earthquake in Istanbul. We compare the performance of the stochastic programming solutions to solutions based on single scenarios and mean values.
\end{abstract}

Mathematics Subject Classification. 90C11, 90C15, 90B06.

Received May 20, 2017. Accepted May 25, 2017.

\section{INTRODUCTION}

The 2004 Indian Ocean tsunami killed more than 225,000 people and dislocated millions of them in countries spread around the Ocean's rim from Kenya to Indonesia. In 1999 hurricane Floyd (CNN [31]), and in 2005 hurricanes Katrina and Rita (TRB [96]) required millions of people to evacuate creating largest traffic jams in the U.S. history. The 2010 Haiti earthquake measured 7.0 magnitude on the Richter scale. It caused a massive level of destruction and imposed tremendous operational challenges on the humanitarian agencies and governments. This resulted in a grim situation: three million affected people, 200,000 deaths, and more than one million wounded (Caunhye et al. [24]). The triple disaster that hit the Tohoku region of Japan on 11 March 2011 triggered a massive human displacement: more than 400,000 people evacuated their homes as a gigantic tsunami induced by a magnitude 9.0 earthquake engulfed the coastal areas, and the following nuclear accident in Fukushima released a large amount of radioactive materials into the atmosphere (Hasegawa [49]).

Natural or man-made disasters such as hurricanes, earthquakes, floods, terrorist attacks impose a serious risk on the humankind. Evacuation of the disaster region is the most predominantly used strategy to protect people threatened by a disaster (Hobeika [50]). Traffic management during an evacuation is critical (Yao et al. [100]) since people's lives are at stake and the unusual surge in traffic demand is generally far beyond the capacity of the road network.

Clearly, the shelter locations affect the time to evacuate a disaster region. Shelters are safe places that protect a population from possible damaging effects of a disaster. They also serve as facilities where evacuees

Keywords. Disaster management, evacuation traffic management, shelter location, constrained system optimal, two-stage stochastic programming, second order cone programming.

1 Department of Industrial Engineering, TED University, Ankara, Turkey.

2 Department of Industrial Engineering, Bilkent University, Ankara, Turkey.

* Correspondant author: hyaman@bilkent.edu.tr 
are provided food, medical care and accommodation. FEMA [40], ARC [2], FEMA [41] and FEMA [43] provide the basis for the design and construction of shelters against different types of disasters. Whether it is built as a new shelter or as a retrofit construction, these preparations require time and need to be done before the disaster takes place. For that reason, the decision of which shelters to open are often made before a disaster occurs.

Disasters are uncertain events: it is not easy to anticipate when and where they will occur and with how much impact. As a result, evacuation planning must be done without exact or complete information. Related studies and real-life practices show a significant uncertainty regarding the evacuation demand due to the unpredictability of time, place and severeness of a disaster and human behavior as well as the changing roadway infrastructure as a result of disaster impacts. When preparing for a disaster, it is very difficult to predict the evacuation demand accurately. However, an accurate knowledge of threat and possible human responses to it is the first guideline that preparedness planning should be based upon (Perry and Lindell [82]). Key studies in the literature (Baker [4], Dash and Gladwin [36], Kim and Oh [57], Mawson [70], Murray-Tuite and Wolshon [74], Perry [81]) state that evacuee behavior is affected by many factors that causes uncertainty in the evacuation demand. We refer the reader to Bayram [7] for a detailed review of uncertainty in evacuation demand caused by human behavior. Although the number of evacuees during Hurricane Rita from the Galvestan and Harris Counties was predicted to be less than 700,000, the actual number turned out to be 1.8 million people (Lindell and Prater [65]). It is also highly probable that the traffic network will be affected and lose capacity in case of a hurricane because of flood. Road segments in the traffic network can be blocked by debris (Berktaş et al. [11], Çelik et al. [25], FEMA [42]) generated after a hurricane (Luther [67]) or by debris caused by collapsed buildings or landslides in an earthquake. They can also be damaged completely or partially in an earthquake, viaducts and bridges may collapse or become unusable. The 1985 Mexico City earthquake wiped out approximately 70 percent of the city's central transportation network (Ardekani and Hobeika [3]). Likewise, the predetermined shelter sites can be affected.

Our aim is to propose a scenario-based evacuation planning model that optimally locates shelter sites and assigns evacuees to the nearest shelter sites and to shortest paths (shortest geographical distance, shortest free flow travel time or shortest congested time) to those shelter sites within a given degree of tolerance to minimize the expected total evacuation time. We focus on this objective rather than minimizing maximum latency as we already impose constraints on the lengths of routes that can be used. We extend the study by Bayram et al. [8] where it is assumed that all parameters are known with certainty.

As we discuss in Section 2, there is a rich literature regarding evacuation planning and management studies. However, most of these studies employ deterministic approaches where only a single hazard scenario is considered, such as the most probabilistic one. On top of that, the number of studies that simultaneously and strategically consider shelter location decisions along with the assignment of evacuees to shelters and to routes is limited. None of these studies take into account the uncertainty in evacuation demand, the road network structure and the shelters simultaneously. Further, majority of the existing studies in the literature employ unrealistic traffic assignment approaches such as user equilibrium or system optimal. There are few studies that consider congestion on the road network and that try to combine the requirements from a system optimal approach of an evacuation management authority and the behaviors of the evacuees. Such studies are only able to manage that through use of bi-level models which can generally be solved through heuristics.

Unlike these studies in the literature, our model considers the uncertainty about future realizations of the evacuation demand, the disruption in the road network and degraded road capacities and disruption of the shelter sites simultaneously. It is a two-stage stochastic nonlinear mixed integer programming model that compromises the perspective of an evacuation planning/management authority and that of the evacuees. We solve practical size problems exactly and in reasonable times by representing the nonlinear objective function with second order cone programming.

Furthermore, we present a real world case study for evacuation planning of a potential major earthquake in Istanbul, Turkey. In our case study the population at risk is evacuated against the possible impact of aftershocks and the secondary disasters following the major earthquake. 
Finally, we carry out extensive numerical testing on the case study to assess the effectiveness of the stochastic programming solutions comparing them with the results from wait and see solutions, where it is assumed that one has perfect information for every possible scenario and mean value solutions in terms of robustness and efficiency. We use criteria such as the expected total evacuation time, maximum regret, expected value of perfect information, value of stochastic solution, and performance measures such as the total evacuation time, maximum latency and percentage of evacuees reaching safety up to a specified time $T$, when both shelters are uncapacitated and capacitated. Maximum regret is the maximum difference between the total evacuation time of a given strategy in a scenario and minimum total evacuation time obtained in that scenario. Expected value of perfect information is the difference between the expected total evacuation time obtained by solving the deterministic problem for every scenario with perfect information and the optimal value obtained using the stochastic optimization solution. And maximum latency is the travel time of the vehicle that stays in the road network longest; it is also the time the evacuation network is cleared off vehicles as we assume that every vehicle enters the network at the same time. We observe that stochastic programming provides robust and effective solutions compared to models based on a single scenario and its superiority is emphasized when shelters are capacitated.

The rest of the paper is organized as follows. In Section 2 we present a literature review on evacuation planning focusing on stochastic location-allocation problems. In Section 3 we describe the problem and give a two-stage stochastic nonlinear mixed integer programming formulation. We present the results of our case study in Section 4 and conclude in Section 5.

\section{Literature REVIEW}

\subsection{Traffic assignment models}

We first review the traffic assignment models. These models differ mainly in their assumed driver behaviors. The common models are the (Stochastic) User Equilibrium ((S)UE, also known as User Optimal), the System Optimal (SO) and the Nearest Allocation (NA) models.

The SUE or UE approaches are not realistic to plan an evacuation during a disaster for the following reasons. The SUE approach assumes that the evacuees choose shortest travel time path depending on their perception of the travel time. The UE approach is a special case of the SUE approach when the variance of travel time perception by the evacuees is zero. For both SUE and UE, the goal of the evacuees is to minimize their individual travel times. The UE assumes that evacuees have full information on travel times on every possible route and that they are able to find the optimal routes (Sheffi [87]). Since evacuees act selfishly to minimize their individual travel times, the UE does not necessarily minimize the total evacuation time in the system. In addition, the assumptions may not be valid in case of a disaster (Galindo and Batta [45]), since the traffic demand is unusual and it is difficult for evacuees to choose the minimum latency routes using their past experiences (Pel et al. [79]).

Evacuation management authority on the other hand considers the benefit of evacuees as a whole and aims to evacuate everyone to safety as soon as possible by minimizing the total evacuation time. Such a traffic assignment plan is called SO. In an SO solution, some travelers may be allocated to routes that are much longer than the shortest ones they would take. Asking evacuees to accept such routes in a situation where they would like to leave the risk zone and reach safety at a shelter as soon as possible may not be reasonable.

In the NA model, each evacuee uses a shortest path based on length (geographical distance) or free flow travel time to reach the nearest shelter. This may be a reasonable approach since during an evacuation, the information on path lengths or free flow travel times is more accessible to evacuees compared to actual travel times. However, such a traffic assignment may lead to poor system performance.

Jahn et al. [55] define the notion of Constrained System Optimal (CSO), a traffic assignment model developed for a route guidance system. This model honors the individual needs by imposing additional constraints to ensure that drivers are assigned to "acceptable" paths only. They define the normal length of a path, as either its free flow travel time, its traversal time in UE, i.e., the congested travel time or its geographic distance. 


\subsection{Deterministic evacuation planning models}

Despite the fact that evacuation planning is typically characterized by great uncertainties, the studies in the literature (Alçada-Almeida et al. [1], Bayram et al. [8], Bish et al. [19], Bretschneider [20], Bretschneider and Kimms [21], Chiu et al. [30], Coutinho-Rodrigues et al. [33], Cova and Johnson [34], Hamacher and Tjandra [48], Kalafatas and Peeta [56], Kongsomsaksakul et al. [59], Sherali et al. [89], Sheu and Pan [90], Tüydeş [97], Yamada [99]), mostly rely on deterministic models.

Bayram et al. [8] propose a deterministic CSO (DCSO) model that optimally locates shelters and that assigns evacuees to the nearest shelters and to shortest paths (in terms of geographical distance, free flow travel time or congested travel time) to those shelters -shortest and nearest within a given degree of tolerance- to minimize the total evacuation time. The solution of their model evacuates the disaster region as quickly as possible, with an efficient but "fair" assignment of evacuees to shelters and to routes. They observe that the SO solution may have unacceptable levels of unfairness whereas the solution in which the evacuees travel to the nearest shelter using a shortest path may result in substantial deterioration in the system performance. Even a small level of tolerance on the side of the evacuees improves the system performance. Overall, they conclude that the location and the number of shelters opened drastically affect the evacuation plan and for a carefully selected number of shelters and tolerance level, an efficient yet fair evacuation plan can be achieved.

These models are deterministic models that adopt a hazard scenario such as worst-case or most probable scenario to plan for locations of shelters and/or evacuation of the endangered population. Our study extends the study by Bayram et al. [8] by incorporating uncertainty.

For a more detailed review of deterministic evacuation planning studies, we refer the reader to Bayram et al. [8] and Bayram [7].

\subsection{Uncertainty in facility location}

The stochasticity regarding the demand, road capacities, facilities and other factors have been widely studied in classical facility location literature and the location literature for disaster management. Frank [44], Cooper [32], Louveaux [66], Laporte et al. [61], Chan et al. [26], Berman and Krass [13], Daskin et al. [37], Berman and Wang [17], Chang et al. [27], Berman et al. [12], study the uncertainty in demand in classical facility location problems and Belardo et al. [10], Psaraftis et al. [83], Wilhelm and Srinivasa [98], Barbarosoğlu and Arda [6], Chang et al. [27], Balçık and Beamon [5], Rawls and Turnquist [84], Mete and Zabinsky [71], Duran et al. [38] and Noyan [78] in disaster management.

Nel and Colbourn [75], Eiselt et al. [39] take into consideration the uncertainty regarding the disruption and/or degradation of edges. Barbarosoğlu and Arda [6], Rawls and Turnquist [84], Günneç and Salman [47] and Noyan [78] propose stochastic models employing similar uncertainty issues in humanitarian relief and disaster management problems.

Mirchandani and Odoni [72], Mirchandani and Oudjit [73], Berman and Odoni [16], Ingolfsson et al. [54] work on facility location networks using stochastic edge lengths or travel times. Mirchandani and Odoni [72] employ a nonlinear utility function for the random edge length. Mete and Zabinsky [71] take into account this same notion in their medical supply location and distribution model they develop for disaster management.

The effects of facility disruption has been studied to a large extent in the classical facility location literature (Bundschuh et al. [22], Snyder and Daskin [91], Snyder et al. [93], Berman et al. [14], Snyder and Daskin [92], Berman et al. [15], Cui et al. [35], Li and Ouyang [63], and Lim et al. [64], Huang et al. [51], Peng et al. [80]) and in disaster management (Rawls and Turnquist [84], Noyan [78]).

To the best of our knowledge, the only study in location literature that takes into consideration the congestion on the road segments is by Chang and Wang [28] for site selection of solid waste facilities.

\subsection{Uncertainty in evacuation planning}

As for the studies taking into account uncertainty in the evacuation literature, most focus on demand uncertainty (Rui et al. [85], Yao et al. [100], Huibregtse et al. [52], Ng and Waller [77], Yazıcı and Özbay 
[101], Kulshrestha et al. [60]) and/or capacity (Shen et al. [88], Ng and Waller [77], Yazıcı and Özbay [101]). Shen et al. [88] develop scenario-based, stochastic, bilevel models that minimize the maximum UE travel time among all node shelter pairs by locating shelters at the upper level and assigning evacuees to shelters and routes in a UE manner at the lower level. Their model is an $\alpha$-reliable mean-excess regret model in the context of the p-median problem Chen et al. [29] in which the distance between the demand nodes and the shelter sites as well as the demand are taken as uncertain parameters. To solve this first model they present a genetic algorithm based approach. The second model is proposed as a real time decision making tool for evacuations and a simulation based approach that uses successive shortest path algorithm is developed to solve it. Stepanov and Smith [94] suggest a multi-objective integer programming formulation for optimal route assignment that employs an $M / G / c / c$ state dependent queuing model to represent congestion and link travel times on links using random arrival rates. Yao et al. [100] consider the evacuation on a network under demand uncertainty. They model a deterministic LP model based on a modified CTM in which they introduce a measure called, coefficient of threat level, which is an estimate of susceptibility of an area to disaster at a particular time and by means of which they can capture the spatio-temporal priorities during evacuation. By focusing on this coefficient of threat level, they develop a robust optimization model. Huibregtse et al. [52] optimize evacuation measures to increase the efficiency of an evacuation plan by considering uncertainty in demand, the behavior of people and the hazard (location, time, and intensity). Ng and Waller [77] present an evacuation planning model that considers demand and capacity uncertainty. They provide a framework that determines the amount of demand inflation (evacuation demand exceeding the planned number) and supply deflation (degradations in road capacities) necessary to ensure a user-specified reliability level. Yazıcı and Özbay [101] propose a SO DTA formulation with probabilistic constraints that takes into account uncertainties in demand and roadway capacities. The model they propose is a CTM-based SO DTA formulation that uses chance constrained programming. They assume that demand and capacity distributions follow discrete probability distributions. They analyze the effects of probabilistic roadway capacities on clearance and average travel times, and the spatial shelter utilization is discussed in terms of shelter management. Their model does not endogenously select the number and location of shelter sites, rather they do sensitivity analysis. Kulshrestha et al. [60] develop a robust bi-level model that considers demand uncertainty and minimizes the total cost to establish and operate shelters at the upper level while assigning evacuees to shelters and routes in a UE manner at the lower level. They confine the uncertain demand to an uncertainty set and determine the optimal locations and capacities of shelters from the worst case scenario that can be realized from this set. Their model is formulated as a mathematical program with complementarity constraints and is solved by an approximation-based cutting plane algorithm, for a total of three (nominal, low and high) demand scenarios.

To the best of our knowledge, there is only a single study that consider the disruption in shelters in the evacuation literature. Li et al. [62] propose a scenario based location model for identifying a set of shelter locations that are robust for a range of hurricane events, by considering disruption in shelter sites. Their model is a DTA-based stochastic bi-level programming model in which at the two-stage stochastic upper level, the central authority selects the shelter sites for a particular scenario. The objective of the upper-level problem is to minimize the weighted sum of the expected unmet shelter demand and the expected total network travel time. In the lower level, evacuees choose their routes in a dynamic UE manner. They develop heuristic algorithms based on Lagrangian relaxation and present a case study for the state of North Carolina for 33 hurricane scenarios.

These studies propose bi-level models which are hard to solve. To solve these problems, heuristic methodologies are developed by Shen et al. [88] and $\mathrm{Li}$ et al. [62] and an approximation based methodology is employed by Kulshrestha et al. [60]. Shen et al. [88] take into account only the uncertainty in capacity, Kulshrestha et al. [60] only consider the uncertainty in demand, and Li et al. [62] only the uncertainty in shelter sites. They employ a UE traffic assignment.

Our model differs from these models and contributes to the literature in that it is a single level model that considers the uncertainty in demand, road network structure and the disruption in shelters simultaneously. It includes the fairness considerations for evacuees. Unlike the solution methodologies employed in the above mentioned studies, we employ an exact solution methodology to solve our problem. 


\subsection{Our contribution}

In this study, we propose a two-stage stochastic scenario-based evacuation planning model. Against this background, the contributions of our study are the following: We introduce a novel model that decides simultaneously on the locations of shelters and the allocations of evacuees to shelters and routes under uncertainty. The model incorporates evacuees' preferences and fairness considerations by routing the evacuees on paths that are not much longer than the shortest paths to the nearest shelters. Unlike the studies in the literature, the model aims to achieve a compromise between evacuation planning authority and the evacuees.

Our study differs from the model by Bayram et al. [8] in that we use a stochastic programming approach, and hence instead of planning the evacuation based on a single hazard scenario, our model considers a range of hazard scenarios. To the best of our knowledge, our model is the first model in the evacuation literature that considers the uncertainty in the evacuation demand, the disruption in the road network, degradation in road capacities and disruption of the shelters simultaneously. This is important, since we are adopting a constrained system optimal approach for the evacuation of the population, the evacuation problem we are dealing with is NP-Hard and it gets harder as it is transformed from a solely assignment problem into a network design problem, i.e., including shelter location decisions increases the complexity of an already NP-Hard problem. For that reason, most of the evacuation models that consider the optimal selection of shelter sites and that also take into account the congestion effect are bi-level models. On top of that, including uncertainty regarding the shelters render the problem even harder.

Further, we investigate a case study with real data about a pending earthquake in Istanbul, Turkey. We report the results of the case study and show that the solution of our stochastic model leads to a significant decrease in the total evacuation time compared to the deterministic and mean value solutions, and planning according to a single scenario can lead to very poor performance. We also analyze the impact of having capacitated shelters on performance measures.

\section{Problem Description AND MOdEL FORMUlation}

We base our work on the DCSO model by Bayram et al. [8] and introduce a two-stage stochastic CSO model, that we refer to as SCSO. We develop our model as an "expected value model", which is the predominant approach in the stochastic programming literature (Sen [86]).

Our model decides on the number and locations of $p$ shelters and the assignment of evacuees to shelters and routes so that the region is evacuated as quickly as possible. The problem is defined on a directed network $G=(N, A)$, where $N$ is the set of nodes and $A$ is the set of arcs (road segments) in the network.

We define the travel time spent on a given road segment as a positive and monotonically increasing function of traffic flow, since an increase in traffic volume will normally decrease the travel speed due to congestion and hence increase the travel time along the road segment.

Bureau of Public Roads (BPR) function is a convex, nondecreasing and differentiable function that expresses the relationship between travel time (or speed) and the volume of traffic on a road segment:

$$
t(x)=t^{0}\left(1+\alpha\left(\frac{x}{c}\right)^{\beta}\right)
$$

where $t(x)$ is the travel time at which assigned volume $x$ can travel on the road segment, $c$ is the practical capacity (maximum flow rate) and $t^{0}$ is the base travel time or free flow travel time at zero volume. The parameters $\alpha \geq 0$ and $\beta \geq 0$ are the tuning parameters defined in accordance with the road characteristics and they are taken as 0.15 and 4 by the U.S. Department of Commerce Bureau of Public Roads, respectively (TAM [95]). $\alpha$ is a parameter that specifies the ratio of free-flow travel time to the travel time at capacity. Parameter $\beta$ determines the steepness of the function, i.e., it specifies how rapidly travel time increases from the free-flow travel time. Among the evacuation planning literature, the studies that employ BPR function to represent the speed-flow relationship in a given road segment (Sherali et al. [89], Kongsomsaksakul et al. [59], Li et al. [62], $\mathrm{Ng}$ and Waller [77]) all use the same $\alpha$ and $\beta$ parameters, i.e., $\alpha=0.15, \beta=4$. For the reasons discussed above we also have employed the same values for these parameters. 
Each $\operatorname{arc} a$ is associated with a convex travel time function (BPR function) $t_{a} . O$ is defined as the set of origin (demand) nodes from where the evacuees at risk are to be evacuated and $F$ as the set of destination nodes (potential shelter sites) where evacuees reach to safety, $O$ and $F$ being disjoint subsets of $N$. The number $p$ is a predetermined parameter that restricts the number of shelter sites that can be opened due to budgetary and/or management issues.

When dealing with uncertainty it is important to consider what happens before (first-stage) and after (secondstage) the uncertainty is revealed (Birge and Louveaux [18]). Considering the evacuation problem in the framework of two-stage stochastic programming, the first stage is about where to locate the shelters before a disaster takes place, and given the location of shelters and realization of the evacuation demand and the disruption in the infrastructure, the second stage assigns evacuees to shelters and to routes.

We define $\Omega$ as the set of disaster scenarios and denote with $p(\omega)$ the probability that disaster scenario $\omega \in \Omega$ occurs. We define $F(\omega) \subseteq F$ as the set of potential shelter sites that are not disrupted in scenario $\omega \in \Omega$. Likewise, $A(\omega) \subseteq A$ is the set of arcs (road segments) that are not disrupted in scenario $\omega \in \Omega$. The binary variable $y_{s}$ is 1 if a shelter site is opened at node $s \in F, 0$ otherwise. Let $P_{r s}(\omega)$ be the set of alternative paths for the origin-destination pair $(r, s)$, in scenario $\omega \in \Omega$. The amount of demand at origin $r \in O, w_{r}(\omega)$, is the number of passenger vehicles that will be evacuated in scenario $\omega \in \Omega$.

We model the tolerance of evacuees using parameter $\lambda$. An evacuee can be persuaded to take a path whose length is at most $(1+\lambda)$ times the length of a shortest path to the closest shelter in a given scenario. The set of acceptable paths from origin $r$ to destination $s$ of tolerance level $\lambda$ in a given scenario $\omega \in \Omega$ is defined as: $P_{r s}^{\lambda}(\omega)=\left\{\pi \in P_{r s}(\omega): d^{\pi} \leq(1+\lambda) d_{r s}^{*}(\omega)\right\}$, where $d^{\pi}$ is the length of path $\pi$ and $d_{r s}^{*}(\omega)$ is the length of a shortest path from $r$ to $s$ in scenario $\omega \in \Omega$. This set is computed using an algorithm developed by Byers and Waterman [23]. The algorithm by Byers and Waterman [23] combines the depth-first search with stacking techniques of theoretical computer science and principles from dynamic programming to list all near-optimal solutions.

In our model, we also use the following decision variables: $v_{\pi}(\omega)$ is the fraction of origin $r$ 's demand that uses path $\pi \in P_{r s}^{\lambda}(\omega)$ from origin $r \in O$ to destination $s \in F(\omega)$ in scenario $\omega \in \Omega$ and $x_{a}(\omega)$ is the amount of traffic, i.e., the number of vehicles on arc $a \in A(\omega)$ in scenario $\omega \in \Omega$.

SCSO is formulated as follows:

$$
\begin{array}{ll}
\min \sum_{\omega \in \Omega} p(\omega) \sum_{a \in A(\omega)} t_{a}^{0}\left(1+\alpha\left(\frac{x_{a}(\omega)}{c_{a}(\omega)}\right)^{\beta}\right) x_{a}(\omega) & \\
\text { s.t. } \sum_{s \in F} y_{s} \leq p, & \forall r \in O, \omega \in \Omega, \\
\sum_{s \in F(\omega)} \sum_{\pi \in P_{r s}^{\lambda}(\omega)} v_{\pi}(\omega)=1 & \forall r \in O, \omega \in \Omega, s \in F(\omega), \\
\sum_{\pi \in P_{r s}^{\lambda}(\omega)} v_{\pi}(\omega) \leq y_{s} & \forall r \in O, \omega \in \Omega, i \in F(\omega), \\
\sum_{s \in F(\omega)} \sum_{\pi \in P_{r s}^{\lambda}(\omega): d^{\pi}>(1+\lambda) d_{r i}^{*}(\omega)} v_{\pi}(\omega)+y_{i} \leq 1 & \forall \omega \in \Omega, a \in A(\omega), \\
x_{a}(\omega)=\sum_{r \in O} \sum_{s \in F(\omega)} w_{r}(\omega) v_{\pi}(\omega) & \forall \omega \in \Omega, \pi \in \bigcup_{r \in O, s \in F(\omega)} P_{r s}^{\lambda}(\omega), \\
v_{\pi}(\omega) \geq 0 & \forall s \in F .
\end{array}
$$


Objective function (3.1) is equal to the expected total evacuation time. Constraint (3.2) limits the number of shelter sites open to a pre-specified number $p$. Constraints (3.3) ensure that all demand is evacuated in each scenario. Constraints (3.4) forbid assigning demand to non-open shelter sites for each scenario. Constraints (3.5) ensure that if the shelter at site $i$ is open and functioning in scenario $\omega$, then the demand at origin node $r$ cannot be routed on any path whose length is longer than $(1+\lambda) d_{r i}^{*}(\omega)$. Let $i_{r}$ be the shelter that is open and that is closest to origin $r$. Due to constraints (3.5), the demand at node $r$ cannot be routed on a path whose length is more than $(1+\lambda) d_{r i_{r}}^{*}(\omega)$. As $i_{r}$ is the closest shelter to $r$ and $d_{r i_{r}}^{*}(\omega)$ is the length of a shortest path from $r$ to $i_{r}$ in scenario $\omega$, these constraints forbid the use of paths whose lengths are longer than $(1+\lambda)$ times the length of the shortest path. Constraints (3.5) for open shelters $i$ other than $i_{r}$ are dominated by constraints (3.5) for shelter $i_{r}$. As we do not know, which shelters are open, we need to include these constraints for all possible shelters. Finally, constraints (3.6) compute the traffic on every arc in each scenario and constraints (3.7) and (3.8) are variable restrictions.

DCSO is a special case of the SCSO model in which $|\Omega|=1$ and Bayram et al. [8] show that DCSO is NP-hard even when $\alpha=0$ and $G$ is bipartite. We also note that our model generalizes the classical facility location model in that the arc costs of our model are nonlinear, we assign the customers (evacuees) to facilities (shelters) and to paths to those facilities. Our model also generalizes the SO and NA traffic assignment approaches. When $\lambda=0$ we have the NA model. When $\lambda=\infty$, we obtain a model for the SO traffic assignment and hence generalize the model given in Sherali et al. [89].

The shelter location decisions $y$ are first-stage (design) variables and are taken in the presence of uncertainty about future realizations. In the second stage, the uncertainty is revealed and recourse decisions $v$ are taken. However, while making the first-stage shelter location decisions, their effect on the second stage assignment decisions and total evacuation time is taken into account. The future effects of shelter location decisions is measured by taking the expectation of the recourse function on possible scenarios. Because $y$ is a binary variable, our model has a $0-1$ first stage problem. In addition, since the objective function of the second stage is nonlinear, the model has a nonlinear second stage problem.

We reformulate the SCSO as a second order conic mixed integer programming model as done in Bayram et al. [8]. Consider an inequality of the form,

$$
r^{2^{l}} \leq s_{1} s_{2} \ldots s_{2^{l}} \text { for } r, s_{1}, s_{2}, \ldots, s_{2^{l}} \geq 0 .
$$

An equivalent representation of the inequality (3.9) can be achieved by using $O\left(2^{l}\right)$ variables and $O\left(2^{l}\right)$ hyperbolic inequalities of the form

$$
u^{2} \leq v_{1} v_{2}, u, v_{1}, v_{2} \geq 0
$$

as shown by Nemirovski and Tal [76]. Then each hyperbolic inequality can easily be transformed into a secondorder cone inequality

$$
\left\|2 u, v_{1}-v_{2}\right\| \leq v_{1}+v_{2}
$$

We first define auxiliary variables $\mu_{a}(\omega)$ for each $a \in A(\omega), \omega \in \Omega$ and move the nonlinearity from the objective function to the constraints. The objective function of the SCSO model becomes:

$$
\sum_{\omega \in \Omega} p(\omega) \sum_{a \in A(\omega)}\left(t_{a}^{0} x_{a}(\omega)+\frac{t_{a}^{0} \alpha}{c_{a}(\omega)^{\beta}} \mu_{a}(\omega)\right)
$$

and we add the constraints $x_{a}(\omega)^{\beta+1} \leq \mu_{a}(\omega)$ for all $\omega \in \Omega, a \in A(\omega)$. 
We take $\beta=4$ and represent $x_{a}(\omega)^{5} \leq \mu_{a}(\omega)$ with

$$
\begin{aligned}
& x_{a}(\omega)^{2} \leq \theta_{a}(\omega) h_{a}(\omega), \\
& \theta_{a}(\omega)^{2} \leq u_{a}(\omega) x_{a}(\omega), \\
& u_{a}(\omega)^{2} \leq \mu_{a}(\omega) x_{a}(\omega) \\
& h_{a}(\omega)=1, \theta_{a}(\omega), u_{a}(\omega), x_{a}(\omega), \mu_{a}(\omega) \geq 0 .
\end{aligned}
$$

These hyperbolic inequalities can be represented with second order conic inequalities presented below:

$$
\begin{aligned}
& \left\|2 x_{a}(\omega), \theta_{a}(\omega)-h_{a}\right\| \leq \theta_{a}(\omega)+h_{a}, \\
& \left\|2 \theta_{a}(\omega), u_{a}(\omega)-x_{a}(\omega)\right\| \leq u_{a}(\omega)+x_{a}(\omega) \\
& \left\|2 u_{a}(\omega), \mu_{a}(\omega)-x_{a}(\omega)\right\| \leq \mu_{a}(\omega)+x_{a}(\omega) \\
& \theta_{a}(\omega) \geq 0, h_{a}=1, u_{a}(\omega) \geq 0, x_{a}(\omega) \geq 0, \mu_{a}(\omega) \geq 0 .
\end{aligned}
$$

The resulting model is solved using an off-the-shelf solver.

\section{An illustrative CaSe StUdy: evacuation of istanbul City AFter a Potential EARTHQUAKE}

\subsection{Risk of aftershocks and secondary disasters}

A study by Marano et al. [69] presents a quantitative and geospatial description of global losses due to earthquake-induced secondary disasters, including landslide, liquefaction, tsunami and fire, for events that took place through 1968-2008. Marano et al. [69] state in their study that $21.5 \%$ of fatal earthquakes have deaths due to secondary causes. In this study, Turkey is shown among the countries where tsunamis, landslides, and fires have been observed as secondary disasters following a major earthquake.

The 1999 Marmara (Turkey) earthquake that devastated the cities of Sakarya, Izmit and Yalova initiated the efforts for earthquake preparedness and response planning for a potential major earthquake in Istanbul. The Istanbul Metropolitan Municipality (IMM) started a disaster prevention and mitigation study that was conducted in collaboration with the Japan International Cooperation Agency (JICA) IMM-JICA [53]. Following the potential major earthquake in Istanbul, aftershocks as observed during the 1999 Marmara earthquake and different secondary disasters such as landslides, floods, fires and possibly tsunamis can impact different geographical areas of Istanbul city and can cause further fatalities. In the technical report by IMM and JICA it is stated that there does not exist an emergency evacuation system in Istanbul, Turkey and that it is imperative that a community evacuation system be established in order to mitigate and minimize human casualties due to second or third aftershocks and secondary disasters following the earthquake. For that reason, the case study we present here assumes an emergency evacuation in the sense that we are evacuating people to protect them from the impact of aftershocks and the secondary disasters.

\subsection{Scenario and data preparation}

In coordination with scientific institutions and researchers, IMM-JICA study determines four scenarios for the earthquake, scenarios A, B, C and D. Scenario A is referred to as the most probable scenario and scenario $\mathrm{C}$ as the worst case scenario. We consider 12 scenarios that correspond to different severeness of the disaster. In the IMM-JICA report, the evacuation demand specific to each origin node is only given for scenario A in detail, and the information for other scenarios is not disclosed. For that reason, our base (nominal) scenario is scenario A. By taking into account the length of the fault line that is predicted to be affected and the magnitude of the earthquake in scenarios B, C and D compared to those of Scenario A, we specify the values of arc and shelter disruption probabilities for risk zones and the evacuation demand at each origin node for these scenarios. We 
generate three different scenarios for each of the original scenarios A, B, C and D in the report, which adds up to 12 scenarios in total. Generally, scenarios 2, 6, and 10 represent more destructive earthquake scenarios.

No probability values regarding the scenarios are given in the report. Scenarios 0,4 and 8 represent the original scenario A in our 12-scenario setting. We randomly assign probabilities to each of the 12 scenarios in such a way that the sum of the probabilities for scenarios 0,4 and 8 , which is the probability of scenario $\mathrm{A}$, is highest and the probabilities related to 12 scenarios add up to 1 .

The magnitude of the earthquake is expected to be 7.5, 7.4, 7.7, and 6.9 and the length of the fault line that is expected to be broken is 119, 108, 174, and $37 \mathrm{~km} . \mathrm{s}$ long for scenarios scenarios A, B, C and D respectively. In the report by IMM-JICA [53], it is stated that 1.3 million citizens will require shelters in accordance with the most probable scenario. A similar number for evacuation demand is given by Kırıkçı [58]. The evacuation demand for other scenarios are not explicitly stated in the report by IMM-JICA. As the length of the fault line that is expected to be broken and the expected magnitute of an earthquake increase, the expected damages incurred and the expected evacuation demand will increase. By taking into account the length of the fault line that is predicted to be affected and the magnitude of the earthquake in scenarios B, C and D compared to those of scenario A, we specify the evacuation demand at each origin node for these scenarios.

The report also includes a seismic microzonation that divides the city into smaller disaster regions with respect to a spatial risk analysis that takes into account their seismic characteristics and damage patterns in different scenarios. Peak Ground Acceleration (PGA) is a measure of how hard the earth shakes in a given geographic area. Five different risk zones are determined for scenarios A, B, C, and D in accordance with the PGA distributions. Damage probabilities specific to road segments, bridges and viaducts in each risk zone are determined and potential damage to the road network, infrastructure and buildings are calculated in accordance with the PGA distribution, as well. By taking into account the length of the fault line that is predicted to be affected and the magnitude of the earthquake in scenarios B, C and D compared to those of scenario A, we specify the values of arc and shelter disruption probabilities for risk zones in each of these scenarios. We assign the highest probability of disruption to risk zone 1, the risk zone closest to the fault line, and the lowest one to risk zone 5. We assign a higher probability of disruption to scenarios 2, 6 and 10 related to the original worst case scenario $\mathrm{C}$.

We classify the arcs and potential shelter sites of the original network into sets in accordance with which risk zone they are located in. For a particular scenario, we randomly determine if an arc (road segment) is disrupted by considering the risk zone it is located in and the probability of disruption assigned to this zone. If the arc is disrupted, we again randomly specify how much of its original capacity is degraded in multiples of a single lane capacity. For instance, if a road segment with three lanes having a capacity of 6,000 vehicles per hour is disrupted, it may be degraded to a capacity of 4,000, 2,000 or 0 vehicles per hour.

The Disaster Coordination Center (DCC - AKOM in Turkish) specified 49 potential shelter sites in Istanbul to serve as safe facilities to provide the evacuees with food, accommodation and medical care Kırıkçı [58]. The main criteria used in determining potential shelter sites are accessibility by at least two alternative routes, proximity to major highways, and availability of land (Görmez et al. [46]). Likewise, in accordance with which risk zone a shelter site is located in and the probability assigned to that risk zone, a shelter site may get disrupted in which case it will no longer be able to serve the evacuees. We assign smaller disruption probabilities for shelters as they are fortified structures against earthquakes.

We generated our instances using the data from the IMM-JICA study and DCC. As the European and Anatolian sides of Istanbul are connected by three bridges, we assume that the population living on each side will be evacuated to shelters on their own side. We use the roads given in the IMM-JICA report together with some other smaller capacity roads. We assume that the free flow travel time in a degraded (not totally disrupted) road segment is the same as the one in the original network. Each vehicle is assumed to carry four passengers on the average. Figure 1 illustrates the road network structure, the potential shelter sites and the demand points that we use in our study (the district of Silivri with one demand point and one shelter site is out of the boundaries of Istanbul European Road Network Map). 

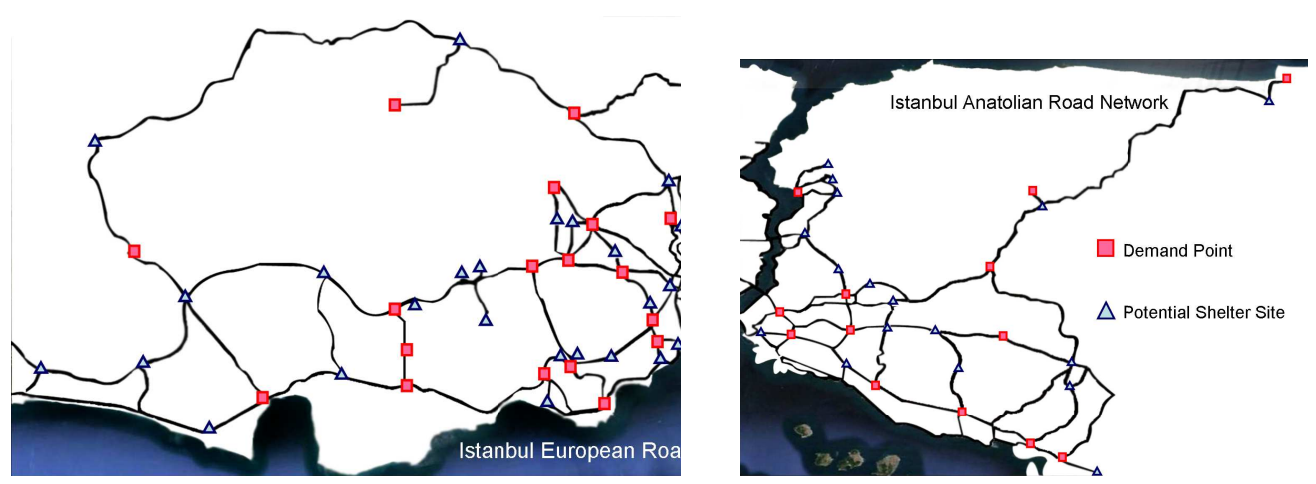

FiguRE 1. Istanbul European and Anatolian road networks, potential shelter sites and demand points.

TABLE 1. Istanbul European instances.

\begin{tabular}{ccccccc}
\hline Scenario & $|N|$ & $|A|$ & Total Demand & $|F|$ & $\begin{array}{c}\text { Probability } \\
\text { of Scenario }\end{array}$ & $\begin{array}{c}\text { Total Road } \\
\text { Network Capacity }\end{array}$ \\
\hline ON & 80 & 238 & 272,900 & 32 & & $1,246,000$ \\
\hline 0 & 80 & 221 & 245,322 & 28 & 0.1461 & $1,098,000$ \\
1 & 80 & 228 & 219,960 & 30 & 0.0754 & $1,128,000$ \\
2 & 80 & 219 & 328,832 & 26 & 0.0436 & $1,074,000$ \\
3 & 80 & 213 & 133,161 & 28 & 0.0872 & $1,022,000$ \\
4 & 80 & 211 & 245,642 & 31 & 0.145 & $1,066,000$ \\
5 & 80 & 220 & 224,481 & 30 & 0.0439 & $1,072,000$ \\
6 & 80 & 223 & 325,988 & 29 & 0.0255 & $1,094,000$ \\
7 & 80 & 218 & 136,819 & 29 & 0.0834 & $1,068,000$ \\
8 & 80 & 221 & 245,622 & 30 & 0.1152 & $1,082,000$ \\
9 & 80 & 217 & 218,378 & 30 & 0.0897 & $1,080,000$ \\
10 & 80 & 223 & 327,002 & 25 & 0.0669 & $1,068,000$ \\
11 & 80 & 224 & 133,883 & 28 & 0.0782 & $1,094,000$ \\
\hline
\end{tabular}

We used the geographical distances as arc lengths in our analysis. Clearly, using geographical distances instead of real travel times as the normal length of a path in modeling evacuees' choices may result in a congested system. However, as pointed out earlier, assuming that the evacuees have full information on travel times on every possible route is not realistic. Instead, one may try to estimate the congested travel times. Our model permits the use of these estimates as normal lengths.

In Tables 1 and 2, we present the properties of our instances. ON is the original network when the road network and the shelters are not disrupted and the demand in ON is the demand of scenario A in IMM-JICA report. And rest of the instances in those tables are scenarios 0 through 11 with the remaining number of arcs, shelters and total road network capacity after the evacuation network is disrupted. We perform our computational tests on a personal computer with a $2.4 \mathrm{GHz}$ Intel i7-3630QM CPU and 16 GB of RAM using Java ILOG CPLEX version 12.5.1.

\subsection{Quality of stochastic programming solutions}

If it were possible to wait until all the uncertainties are revealed and solve the evacuation problem with perfect information in hand for each scenario, this would be called a wait and see solution (WSS) Madansky [68]. On the other hand, one could solve the stochastic problem by replacing all the random parameters with their means, 
TABLE 2. Istanbul Anatolian instances.

\begin{tabular}{cccrrrc}
\hline Scenario & $|N|$ & $|A|$ & Total Demand & $|F|$ & $\begin{array}{c}\text { Probability } \\
\text { of Scenario }\end{array}$ & $\begin{array}{c}\text { Total Road } \\
\text { Network Capacity }\end{array}$ \\
\hline ON & 50 & 146 & 83,133 & 17 & & 748,000 \\
\hline 0 & 50 & 137 & 74,965 & 16 & 0.1461 & 666,000 \\
1 & 50 & 135 & 63,263 & 17 & 0.0754 & 668,000 \\
2 & 50 & 135 & 101,482 & 15 & 0.0436 & 676,000 \\
3 & 50 & 133 & 41,920 & 15 & 0.0872 & 646,000 \\
4 & 50 & 137 & 75,586 & 17 & 0.145 & 690,000 \\
5 & 50 & 140 & 66,203 & 17 & 0.0439 & 676,000 \\
6 & 50 & 136 & 103,708 & 17 & 0.0255 & 688,000 \\
7 & 50 & 140 & 41,599 & 13 & 0.0834 & 700,000 \\
8 & 50 & 136 & 75,299 & 16 & 0.1152 & 678,000 \\
9 & 50 & 143 & 66,451 & 16 & 0.0897 & 724,000 \\
10 & 50 & 136 & 97,111 & 13 & 0.0669 & 644,000 \\
11 & 50 & 131 & 39,559 & 17 & 0.0782 & 642,000 \\
\hline
\end{tabular}

this solution is called the mean value solution (MVS). Expected value of perfect information (EVPI) is the difference between the expected total evacuation times of wait and see solution and the stochastic programming solution and value of stochastic solution (VSS) is the difference between the expected total evacuation times of the stochastic programming and mean value solutions. Expected value of perfect information measures the value of knowing the future with certainty, while value of stochastic solution assesses the value of knowing and using distributions of future outcomes (Birge and Louveaux [18]).

To measure the expected value of perfect information and value of stochastic solution, we solve our evacuation problem first by considering a specific scenario, i.e., employ the wait and see solution. We also solve the evacuation problem by taking the mean of the stochastic parameters in each scenario, which results in the mean value solution. Given the shelter locations from the wait and see solution and the mean value solution for a specific scenario, we solve our problem for each scenario and obtain the total evacuation times in these scenarios separately for both of these solution strategies. We adopt an approach that includes only the shelter location decisions from wait and see solution and mean value solution to solve the model for each scenario in order to compute expected value of perfect information and value of stochastic solution, and show the quality of our stochastic programming solution.

For the mean value solution, the demand for each origin is taken as the mean of the demand values at these origins in all possible scenarios. For the number of potential shelter sites, $|F|$, we take a shelter as disrupted if it is disrupted in two or more scenarios. As for the road segments, if a road segment is disrupted in three or more scenarios it is removed from the graph of mean value scenario, and if this is not the case we take the average of the arc capacities over all possible scenarios to find the capacity of the road segment as a multiple of lanes. This results in having 9 disrupted shelters and 23 disrupted road segments for Istanbul European network and 4 disrupted shelters and 7 disrupted road segments for Istanbul Anatolian network.

\subsection{Uncapacitated Shelters}

In our first experiment, we investigate the effect of the number of shelters $p$ and the tolerance level $\lambda$ on the total evacuation time and the difficulty of the problem when shelters are uncapacitated. We compute the set of feasible paths of tolerance degree $\lambda$ by using the algorithm by [23]. In Table 3, we report the results of the SCSO model for the Istanbul European and Anatolian instances for different values of $p$ and $\lambda$. For each instance, we report the number of shelters opened (\#os), the optimal expected total evacuation time and the solution time in seconds. All instances are solved to optimality and the longest computation time is a little more than one hour. As we increase the level of tolerance the number of feasible paths that the evacuees can be assigned 
TABLE 3. Results for the Istanbul European and Anatolian instances

\begin{tabular}{c|cccc|cccc}
\hline & \multicolumn{3}{c}{ Istanbul European } & \multicolumn{4}{c}{ Istanbul Anatolian } \\
\hline$\lambda$ & $p$ & \#os & Exp. Tot. Evac. Time & Solution Time & $p$ & $\#$ os & Exp. Tot. Evac. Time & Solution Time \\
\hline \multirow{5}{*}{0} & 10 & 10 & $25,448,247$ & 300.60 & 5 & 5 & 78,850 & 72.46 \\
& 20 & 20 & $25,160,535$ & 163.35 & 10 & 10 & 56,621 & 36.61 \\
& 25 & 20 & $25,135,322$ & 199.10 & 13 & 12 & 56,592 & 29.11 \\
& 30 & 20 & $25,135,322$ & 166.04 & 15 & 12 & 56,574 & 26.53 \\
& 32 & 20 & $25,135,322$ & 215.57 & 17 & 12 & 56,574 & 27.86 \\
\hline \multirow{5}{*}{0.1} & 10 & 10 & $10,293,567$ & 2471.33 & 5 & 5 & 42,366 & 97.67 \\
& 20 & 20 & $10,167,674$ & 880.59 & 10 & 10 & 32,306 & 47.48 \\
& 25 & 20 & $10,160,242$ & 934.62 & 13 & 13 & 32,212 & 41.93 \\
& 30 & 20 & $10,160,242$ & 1304.19 & 15 & 13 & 32,202 & 40.69 \\
& 32 & 20 & $10,160,242$ & 755.29 & 17 & 13 & 32,202 & 39.01 \\
\hline \multirow{5}{*}{0.15} & 10 & 10 & $6,432,920$ & 3048.37 & 5 & 5 & 40,913 & 119.37 \\
& 20 & 20 & $6,390,771$ & $2,344.98$ & 10 & 10 & 32,318 & 54.62 \\
& 25 & 20 & $6,385,797$ & 2682.86 & 13 & 13 & 32,218 & 47.10 \\
& 30 & 20 & $6,385,797$ & 3634.32 & 15 & 13 & 32,193 & 46.41 \\
& 32 & 20 & $6,385,797$ & 3522.80 & 17 & 13 & 32,193 & 42.54 \\
\hline
\end{tabular}

increases. And due to this reason, we observe that the expected total evacuation time decreases drastically as the tolerance parameter $\lambda$ increases for both networks. However, an increase in the number of feasible paths means an increase in the number of variables in the model. For that reason, the solution times deteriorates as the tolerance level increases. We observe that, in general, the solution times decrease with increasing $p$ and increase with increasing $\lambda$.

We note here that at most 20 and 13 shelters are opened in Istanbul European and Anatolian instances, respectively, even when $p$ is larger. As also noted in Bayram et al. [8], it is not necessarily beneficial to open more shelters as this may cause some road segments to be extremely congested.

For the case when shelters are uncapacitated, we compare the quality of 15 different solution strategies, named as $0,1, \ldots, 11$, ON, MVS, S. Among these, $0,1, \ldots, 11$ are the optimal solutions corresponding to the original scenarios, ON represents the solution obtained from using the original undisrupted network with the original demand from scenario A in IMM-JICA report, and MVS and S represents the mean value solution and stochastic programming solution, respectively. The regret of a solution in a given scenario is the difference between the total evacuation time for that solution in that scenario and the minimum total evacuation time for that scenario. The maximum regret for a solution is the maximum of its regrets over all scenarios.

In Table 4, we present the total evacuation times for all 15 solutions and 12 scenarios, as well as the expectation of the total evacuation times. We take $\lambda=0.1$ and $p=32$ for the European network and $\lambda=0.2$ and $p=17$ for the Anatolian network. Table 5 gives the wait and see solution, expected value of perfect information and value of stochastic solution for both networks. Finally, in Table 6, we present the regrets of the solutions in all 12 scenarios and the maximum regrets.

It is clearly seen that, the stochastic programming solution provides a much better evacuation planning compared to ON solution. With the stochastic programming solutions, we obtain about 7 and 2 times better results with respect to expectation, compared to the ON solutions for Istanbul European and Istanbul Anatolian networks, respectively. With respect to maximum regret, the results are more striking. Stochastic Programming Solution has almost 70 and 7 times better maximum regret values for Istanbul European and Istanbul Anatolian networks, respectively.

For both Istanbul European and Anatolian networks, the stochastic programming solution performs best in terms of the expected total evacuation time and in terms of the maximum regret. When we use the stochastic 


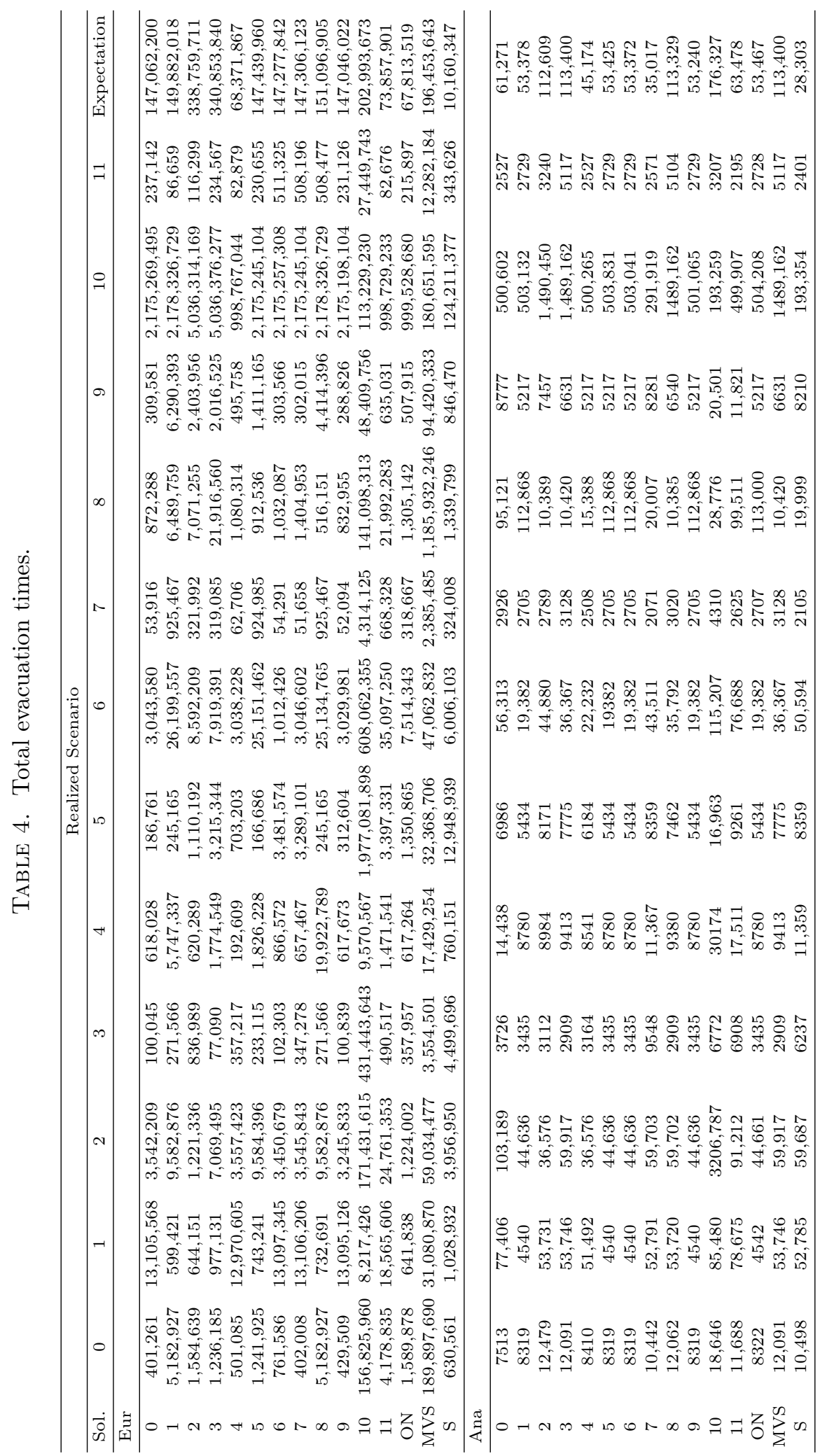


TABLE 5. WSS, EVPI, VSS.

\begin{tabular}{ccc}
\hline & Istanbul European & Istanbul Anatolian \\
\hline WSS & $7,896,033$ & 20,197 \\
EVPI & $2,264,314$ & 8106 \\
VSS & $186,293,296$ & 85,097 \\
\hline
\end{tabular}

programming solution, on the average, a vehicle spends about 10 hours more and 7 minutes more to reach its shelter, compared to what it would have spent if we had perfect information about the disaster for Istanbul European and Anatolian networks, respectively. If we look at a specific scenario, say, if we assume we have perfect information for scenario 10 in Istanbul Anatolian network and if we locate the shelters and make evacuation plans accordingly, and if indeed this scenario realizes, an evacuee will reach a safe shelter about at the same time he/she reaches safety in the stochastic programming solution. The value of stochastic solution is very high, especially for the Istanbul European network. Indeed, the ratio of the expected total evacuation time for the mean value solution to the one of the stochastic programming solution is 19.3 and 4.0 for Istanbul European and Anatolian networks, respectively. We also observe that planning according to a single scenario can lead to very poor performance. For Istanbul European network, if we plan according to scenario 10, the expected total evacuation time is about 20 times higher than the expected total evacuation time for the stochastic programming solution.

We also investigate the performance of the solutions for different values of tolerance levels. We report in Table 7 the total evacuation time versus $\lambda$ for both networks when $p=32$ and $p=17$, respectively for scenario 10. We observe that the total evacuation time for the stochastic programming solution is almost as good as solution 10 for scenario 10. We notice that ON solution tends to give better solutions for small $\lambda$ values compared to mean value solution. Neither ON nor MVS solution is able provide a better total evacuation time compared to stochastic programming solution. The solutions ON and MVS provide are about 3.1 and 33.2 times and 13.2 and 2.4 times worse than that of stochastic programming solution when $\lambda=0$ and $\lambda=0.15$, respectively. For Istanbul Anatolian network these numbers are about 8 and 2.5 when $\lambda=0.1$ and 2.6 and 7.7 when $\lambda=0.2$.

Finally, we use the performance measures "maximum latency (or maximum experienced travel time)", and "percentage of evacuees reaching safety up to a specified time $T$ " to evaluate the performance of our solutions. Table 8 illustrates the maximum latency incurred by the evacuees for scenario 10 of Istanbul Anatolian network, for different tolerance levels, when $p=17$. After solution 10, which is the optimal for the realized scenario, the stochastic programming solution has the least maximum latency, many times better than that of any other solution. For some $\lambda$ values the maximum latency incurred in stochastic programming solution is the same as that of solution 10. In terms of maximum latency, stochastic programming solution provides 4.4 times and 14.6 times better solutions than those of the $\mathrm{ON}$ and mean value solutions, respectively. Figure 2 also depicts the percentage of evacuees reaching safety across time for different strategies, for scenario 10 of Istanbul Anatolian instance, when $p=17$ and $\lambda=0.2$ and for scenario 0 of Istanbul European instance, when $p=32$ and $\lambda=0.15$. Since realized scenario is 10 , solution 10 evacuates $100 \%$ of the population in less than six hours. While the stochastic programming solution evacuates almost everyone to safety within the same time as solution 10 , the original network solution ON and the mean value solution MVS, can only evacuate $85 \%$ and $69 \%$ of the population within the same time. Solution strategy 7 starts really poorly in the beginning but then evacuates everyone within almost the same times as solution strategy 10 and stochastic programming solution. With stochastic programming solution, almost $90 \%$ of evacuees reach safe shelters in less than 5 hours and it takes a little bit more than 5 hours to reach shelters only for less than $10 \%$ of the evacuees and everyone is evacuated to safe shelters in less than 6 hours. The situation is similar for the Istanbul European instance. Stochastic programming solution beats the ON and mean value solutions and most of the other solution strategies. With stochastic programming solution more than $70 \%$ of evacuees reach safety in less than 6.5 hours and only less 


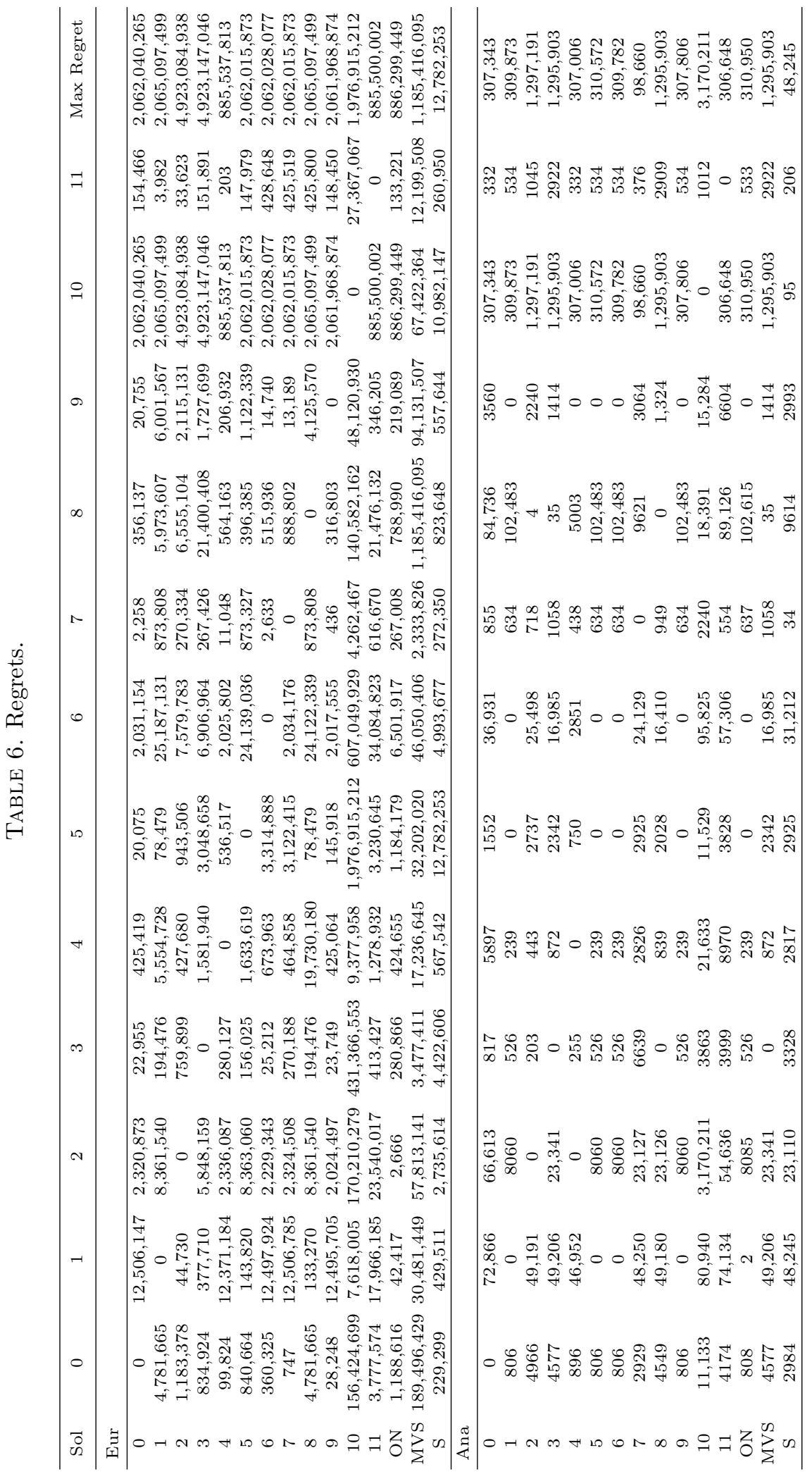


TABLE 7. Total Evacuation Time for various levels of tolerance for scenario 10.

\begin{tabular}{|c|c|c|c|c|c|c|c|c|}
\hline \multirow[t]{2}{*}{$\lambda$} & \multicolumn{8}{|c|}{ Istanbul European } \\
\hline & 0 & 1 & 2 & 3 & 4 & 5 & 6 & 7 \\
\hline 0 & $2,175,259,598$ & $998,806,214$ & $10,728,808,279$ & $10,728,822,320$ & $998,822,735$ & $2,175,259,598$ & $2,175,322,600$ & $2,175,259,598$ \\
\hline 0.1 & $2,175,269,495$ & $2,178,326,729$ & $5,036,314,169$ & $5,036,376,277$ & $998,767,044$ & $2,175,245,104$ & $2,175,257,308$ & $2,175,245,104$ \\
\hline \multirow[t]{2}{*}{0.15} & $2,175,269,190$ & $2,175,198,536$ & $5,036,312,908$ & $2,175,249,906$ & $753,716,960$ & $2,175,269,190$ & $2,175,255,531$ & $2,175,245,104$ \\
\hline & 8 & 9 & 10 & 11 & $\mathrm{ON}$ & MVS & $\mathrm{S}$ & \\
\hline 0 & $2,175,265,854$ & $753,696,271$ & $323,291,760$ & $998,743,086$ & $1,002,780,099$ & $10,728,776,770$ & $323,365,972$ & \\
\hline 0.1 & $2,178,326,729$ & $2,175,198,104$ & $113,203,246$ & $998,729,233$ & $999,528,680$ & $180,651,595$ & $124,211,377$ & \\
\hline \multirow[t]{3}{*}{0.15} & $2,175,198,890$ & $753,632,213$ & $74,770,390$ & $753,679,132$ & $999,527,218$ & $181,278,134$ & $75,875,383$ & \\
\hline & \multicolumn{8}{|c|}{ Istanbul Anatolian } \\
\hline & 0 & 1 & 2 & 3 & 4 & 5 & 6 & 7 \\
\hline 0 & $1,585,374$ & $2,679,390$ & 660,747 & $1,241,800$ & 811,153 & $1,585,374$ & 660,747 & $1,645,442$ \\
\hline 0.1 & 501,214 & 589,880 & $1,577,623$ & 501,214 & 500,721 & $1,583,700$ & $1,588,090$ & 292,361 \\
\hline \multirow[t]{2}{*}{0.2} & 500,602 & 503,132 & $1,490,450$ & $1,489,162$ & 500,265 & 503,831 & 503,041 & 291,919 \\
\hline & 8 & 9 & 10 & 11 & $\mathrm{ON}$ & MVS & $\mathrm{S}$ & \\
\hline 0 & $1,585,370$ & $2,420,442$ & 547,125 & 817,167 & 661,202 & $1,577,324$ & 547,212 & \\
\hline 0.1 & 501,214 & 589,880 & 197,047 & 501,931 & $1,588,809$ & 500,814 & 197,260 & \\
\hline 0.2 & $1,489,162$ & 501,065 & 193,259 & 499,907 & 504,208 & $1,489,162$ & 193,354 & \\
\hline
\end{tabular}

TABLE 8. Maximum Latency for various levels of tolerance for scenario 10, Istanbul Anatolian.

\begin{tabular}{|c|c|c|c|c|c|c|c|c|c|c|c|c|c|c|c|}
\hline$\lambda$ & 0 & 1 & 2 & 3 & 4 & 5 & 6 & 7 & 8 & 9 & 10 & 11 & $\mathrm{ON}$ & MVS & $\mathrm{S}$ \\
\hline 0 & 76.01 & 99.46 & 21.95 & 73.44 & 21.95 & 76.01 & 21.95 & 76.01 & 76.01 & 76.01 & 14.12 & 21.95 & 21.96 & 76.01 & 14.12 \\
\hline 0.05 & 76.01 & 24.77 & 76.01 & 73.44 & 73.44 & 76.01 & 76.01 & 76.01 & 73.44 & 76.01 & 5.18 & 73.44 & 76.03 & 73.44 & 5.18 \\
\hline 0.1 & 21.95 & 21.95 & 76.01 & 21.95 & 21.95 & 76.01 & 76.01 & 5.16 & 21.95 & 21.95 & 5.13 & 21.95 & 76.03 & 21.95 & 5.16 \\
\hline 0.15 & 21.95 & 73.53 & 73.53 & 73.53 & 21.95 & 21.95 & 73.53 & 73.53 & 73.53 & 73.53 & 5.13 & 21.95 & 73.54 & 73.53 & 5.17 \\
\hline 0.2 & 21.95 & 21.95 & 73.53 & 73.53 & 21.95 & 21.95 & 21.95 & 5.13 & 73.53 & 21.95 & 5.03 & 21.95 & 21.96 & 73.53 & 5.03 \\
\hline
\end{tabular}

TABLE 9. Maximum Latency for various levels of tolerance across different scenarios for stochastic programming solution, Istanbul Anatolian.

\begin{tabular}{|c|c|c|c|c|c|c|c|c|c|c|c|c|}
\hline Scen & \multicolumn{3}{|c|}{0} & \multicolumn{3}{|c|}{1} & \multicolumn{3}{|c|}{2} & \multicolumn{3}{|c|}{3} \\
\hline$\lambda$ & $\operatorname{Max}$ & Min & Ave & Max & Min & Ave & Max & Min & Ave & Max & Min & Ave \\
\hline 0 & 0.513 & 0.019 & 0.194 & 4.093 & 0.019 & 0.871 & 1.919 & 0.022 & 0.590 & 1.111 & 0.023 & 0.225 \\
\hline 0.1 & 0.513 & 0.018 & 0.194 & 4.093 & 0.018 & 0.870 & 1.919 & 0.022 & 0.588 & 0.631 & 0.023 & 0.149 \\
\hline 0.2 & 0.513 & 0.018 & 0.140 & 4.093 & 0.018 & 0.834 & 1.919 & 0.022 & 0.588 & 0.631 & 0.023 & 0.149 \\
\hline Scen & \multicolumn{3}{|c|}{4} & \multicolumn{3}{|c|}{5} & \multicolumn{3}{|c|}{6} & \multicolumn{3}{|c|}{7} \\
\hline$\lambda$ & Max & Min & Ave & Max & Min & Ave & Max & Min & Ave & Max & Min & Ave \\
\hline 0 & 0.454 & 0.019 & 0.152 & 0.360 & 0.018 & 0.157 & 4.968 & 0.021 & 1.445 & 0.093 & 0.018 & 0.057 \\
\hline 0.1 & 0.454 & 0.018 & 0.150 & 0.360 & 0.018 & 0.156 & 4.968 & 0.018 & 1.444 & 0.093 & 0.018 & 0.057 \\
\hline 0.2 & 0.454 & 0.018 & 0.150 & 0.360 & 0.018 & 0.126 & 1.851 & 0.018 & 0.488 & 0.088 & 0.018 & 0.051 \\
\hline Scen & \multicolumn{3}{|c|}{8} & \multicolumn{3}{|c|}{9} & \multicolumn{3}{|c|}{10} & \multicolumn{3}{|c|}{11} \\
\hline$\lambda$ & $\operatorname{Max}$ & Min & Ave & Max & Min & Ave & Max & Min & Ave & $\operatorname{Max}$ & Min & Ave \\
\hline 0 & 0.639 & 0.019 & 0.266 & 0.423 & 0.018 & 0.186 & 14.123 & 0.024 & 5.635 & 0.615 & 0.018 & 0.228 \\
\hline 0.1 & 0.639 & 0.018 & 0.266 & 0.423 & 0.018 & 0.156 & 5.162 & 0.024 & 2.031 & 0.146 & 0.018 & 0.078 \\
\hline 0.2 & 0.639 & 0.018 & 0.266 & 0.423 & 0.018 & 0.124 & 5.028 & 0.024 & 1.991 & 0.140 & 0.018 & 0.061 \\
\hline
\end{tabular}

than $7 \%$ of the population is evacuated to shelters in more than 15 hours. Everyone is evacuated to safety in less than 16 hours. In Table 9, we report the maximum, minimum and average latency values for various levels of tolerance across different scenarios for stochastic programming solution. For all of the instances, the ratio of the maximum latency to the average latency is less than 5, and for majority of the instances this ratio is between 2 and 3 . This is due to our fairness considerations as we do not assign the evacuees to paths that are much longer that the shortest one they would take. 
Percentage Evacuated, Scenario 0, Istanbul European

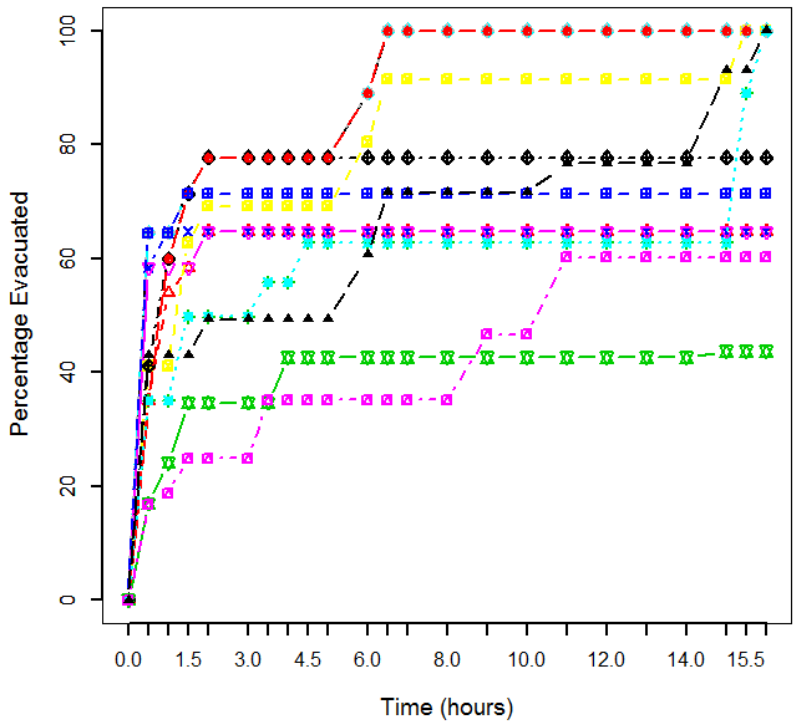

Percentage Evacuated, scenario 10, Istanbul Anatolian

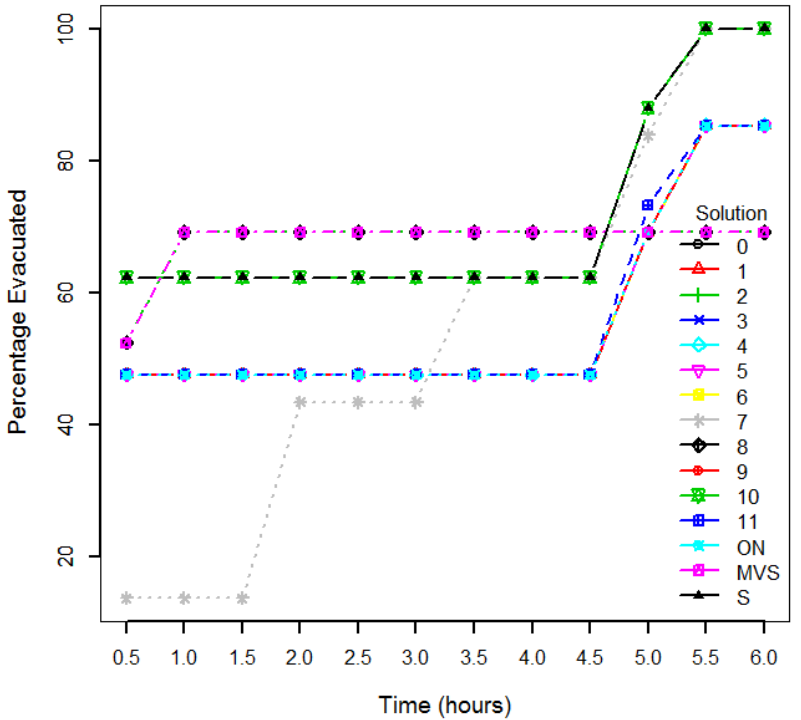

FIGURE 2. The percentage of evacuees reaching safety across time for different strategies.

TABLE 10. Shelter utilization, Istanbul Anatolian.

\begin{tabular}{|c|c|c|c|c|c|c|c|c|c|c|c|c|c|c|c|c|c|}
\hline \multirow[b]{2}{*}{ Scenario } & \multicolumn{17}{|c|}{ Shelters } \\
\hline & 14 & 15 & 16 & 17 & 18 & 19 & 20 & 21 & 22 & 23 & 24 & 25 & 26 & 27 & 28 & 29 & 30 \\
\hline 0 & 1785 & 0 & 0 & 4718 & 1630 & 15,621 & 14,983 & $\mathrm{D}$ & 0 & 12,334 & 0 & 8,517 & 10,316 & 543 & 278 & 0 & 4241 \\
\hline 1 & 1496 & 0 & 0 & 4688 & 1495 & 11,965 & 12,032 & 2,647 & 0 & 10,380 & 0 & 7572 & 6458 & 342 & 276 & 0 & 3912 \\
\hline 2 & 2386 & 0 & $\mathrm{D}$ & 9258 & 0 & 20,264 & 20,968 & 3997 & 0 & 17,382 & 0 & 20,706 & D & 1048 & 352 & 0 & 5122 \\
\hline 3 & 825 & 0 & 0 & D & 3154 & 8306 & 8811 & 7811 & 0 & D & 0 & 0 & 9816 & 0 & 168 & 0 & 3031 \\
\hline 4 & 1414 & 394 & 0 & 4744 & 1839 & 14,798 & 15,532 & 3084 & 0 & 11,936 & 0 & 3847 & 12,862 & 523 & 277 & 0 & 4337 \\
\hline 5 & 1733 & 0 & 0 & 4738 & 738 & 13,568 & 12,956 & 2878 & 0 & 11,204 & 0 & 7434 & 6309 & 395 & 253 & 0 & 3997 \\
\hline 6 & 2512 & 0 & 0 & 5459 & 3280 & 21,039 & 20,908 & 4037 & 0 & 17,221 & 0 & 11,970 & 10,260 & 1523 & 385 & 0 & 5115 \\
\hline 7 & 905 & 0 & $\mathrm{D}$ & 2980 & D & 8919 & 7547 & D & $\mathrm{D}$ & 7126 & 0 & 5259 & 5930 & 0 & 159 & 0 & 2775 \\
\hline 8 & 1755 & 0 & 0 & 4767 & 1898 & 14,597 & 15,110 & 2986 & 0 & 12645 & 0 & 0 & 16,710 & 321 & 269 & 0 & 4242 \\
\hline 9 & 1533 & 0 & 0 & 5659 & 4484 & D & 21,921 & 2563 & 0 & 11,711 & 0 & 7587 & 6442 & 300 & 234 & 0 & 4017 \\
\hline 10 & D & 2512 & 0 & D & 8286 & 28,213 & D & 8888 & 0 & 0 & 0 & D & 36,628 & 7095 & 342 & 0 & 5149 \\
\hline 11 & 951 & 0 & 0 & 3926 & 0 & 7891 & 1895 & 5742 & 0 & 6194 & 0 & 3732 & 4432 & 2247 & 174 & 0 & 2376 \\
\hline Max & 2512 & 2512 & 0 & 9258 & 8286 & 28,213 & 21,921 & 8888 & 0 & 17,382 & 0 & 20,706 & 36,628 & 7095 & 385 & 0 & 5149 \\
\hline Min & 825 & 0 & 0 & 2980 & 0 & 7891 & 1895 & 2563 & 0 & 0 & 0 & 0 & 4432 & 0 & 159 & 0 & 2376 \\
\hline Ave & 1572 & 242 & 0 & 5094 & 2437 & 15,016 & 13,878 & 4463 & 0 & 10,739 & 0 & 6966 & 11,469 & 1195 & 264 & 0 & 4026 \\
\hline
\end{tabular}

In Table 10, we report the utilization numbers of the shelters for Istanbul Anatolian instance with $p=17$ and $\lambda=0.2$ for stochastic programming solution across different scenarios. We also report the maximum, minimum and the average utilization numbers. The utilization number reported in the table is the number of vehicles. Among the 17 potential shelters, i.e., shelters numbered 14 through 30, only 13 are selected to be opened in the optimal solution. The four shelters that are not opened in the optimal solution, i.e., the shelters 16, 22, 24, and 29 can be seen in table, as the utilization numbers in the column under them are all zero. D represents a shelter being disrupted in a specific scenario. As we do not restrict the number of evacuees a shelter can accommodate in the uncapacitated model, we assume that in an optimal solution the opened shelters should be able to hold a capacity of at least as much as the number of evacuees assigned to them. Hence, to be on the safe side with the uncapacitated model, one should prepare shelters with capacities as much as the maximum utilization numbers. 
TABLE 11. Results for the Istanbul European and Anatolian instances when shelters are capacitated.

\begin{tabular}{|c|c|c|c|c|c|c|c|c|c|}
\hline \multicolumn{5}{|c|}{ Istanbul European } & \multicolumn{5}{|c|}{ Istanbul Anatolian } \\
\hline$\lambda$ & $p$ & $\# \mathrm{os}$ & Exp. Tot. Evac. Time & Sol. Time & $\lambda$ & $p$ & $\#$ os & Exp. Tot. Evac. Time & Sol. Time \\
\hline \multirow{4}{*}{0.05} & & & & & \multirow{4}{*}{0.1} & 10 & 10 & 111,695 & 40.42 \\
\hline & 20 & 20 & $22,033,781$ & 111.84 & & 13 & 10 & 111,686 & 44.64 \\
\hline & 25 & 20 & $22,013,994$ & 111.35 & & 15 & 10 & 111,686 & 44.96 \\
\hline & 32 & 20 & $22,013,994$ & 107.43 & & 17 & 10 & 111,686 & 42.55 \\
\hline \multirow{4}{*}{0.1} & & & & & \multirow{4}{*}{0.15} & 10 & 10 & 99,754 & 59.06 \\
\hline & 20 & 20 & $11,600,146$ & 213.45 & & 13 & 10 & 99,720 & 52.22 \\
\hline & 25 & 20 & $11,579,937$ & 224.95 & & 15 & 10 & 99,720 & 52.23 \\
\hline & 32 & 20 & $11,579,937$ & 203.18 & & 17 & 10 & 99,720 & 52.18 \\
\hline \multirow{4}{*}{0.15} & & & & & \multirow{4}{*}{0.2} & 10 & 10 & 87,968 & 47.60 \\
\hline & 20 & 20 & $8,948,507$ & 825.94 & & 13 & 13 & 45,490 & 41.80 \\
\hline & 25 & 20 & $8,939,547$ & 500.99 & & 15 & 13 & 45,490 & 43.33 \\
\hline & 32 & 20 & $8,939,547$ & 652.65 & & 17 & 13 & 45,490 & 43.91 \\
\hline
\end{tabular}

\subsection{Capacitated Shelters}

In our SCSO model, we assume that the shelters have unlimited capacity and that their capacity can be planned according to the allocated demands. In this section, we analyze how fixed capacities affect the quality of stochastic solution.

We add the capacity constraints $\sum_{r \in O} \sum_{\pi \in P_{r s}^{\lambda}(\omega)} w_{r}(\omega) v_{\pi}(\omega) \leq K_{s} y_{s}, \forall \omega \in \Omega, s \in F(\omega)$ where $K_{s}$ is the capacity of shelter $s$. We refer to the resulting model as "stochastic constrained system optimal model with capacitated shelters" and abbreviate it with SCSO-CS. We take shelter capacities as used in Kırıçı [58]. In Table 11, we present the results for the Istanbul European and Anatolian Instances when shelters are capacitated. With original capacities, the problem is infeasible for both Istanbul European and Istanbul Anatolian networks for all $\lambda$ values up to 0.2 . We increase the capacities of some of the shelters so that the problem becomes feasible for some $\lambda$ values. With the updated capacities, the problem is feasible for Istanbul European network for $\lambda \geq 0.05$ and for Istanbul Anatolian network for $\lambda \geq 0.1$.

For the case when shelters are capacitated, we compare the quality of 14 different solutions, named as 0 , $1, \ldots 11$, MVS, S, with $0,1, \ldots 11$ being the optimal solutions corresponding to the original scenarios, MVS and $\mathrm{S}$ representing the mean value solution and stochastic programming solution, respectively.

In Table 12, we present the total evacuation times for all 14 solutions and 12 scenarios, as well as the expectation of the total evacuation times, when shelters are capacitated. We take $\lambda=0.1$ and $p=32$ for the Istanbul European network and $\lambda=0.2$ and $p=17$ for the Istanbul Anatolian network. Table 13 gives the wait and see solution, expected value of perfect information and value of stochastic solution for both networks. Finally, in Table 14, we present the regrets of the solutions in all 12 scenarios and the maximum regrets over all scenarios.

For both Istanbul European and Anatolian networks, there is no single solution other than the stochastic programming solution that is feasible over all possible scenarios, when shelters are capacitated. In Tables 12 and $14, " \infty$ " basically means that the proposed solution is not feasible for that specific scenario. For that reason the stochastic programming solution is superior compared to all other solutions.

When we use the stochastic programming solution, on the average, a vehicle spends about 7 hours more and a little bit more than 11 minutes more to reach its shelter, compared to what it would have spent if we had perfect information about the disaster for Istanbul European and Anatolian networks, respectively. If we look at a specific scenario, say, if we assume we have perfect information about scenario 10, a possible worst case scenario, and if we locate the shelters and make evacuation plans accordingly, and if indeed this scenario realizes, an evacuee will reach a safe shelter at the same time he/she reaches safety in accordance with the stochastic programming solution, in Istanbul European network. 


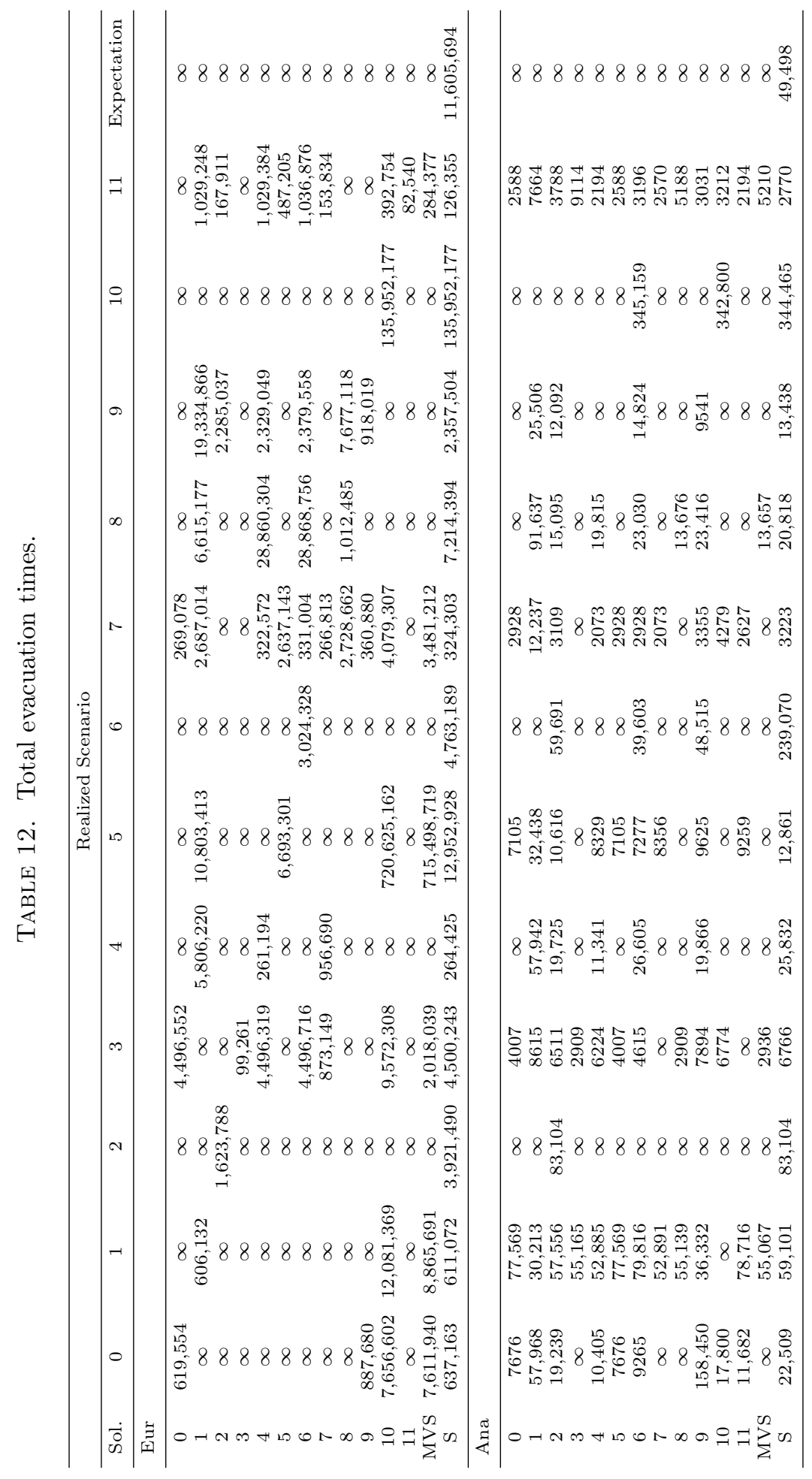


TABLE 13. WSS, EVPI, VSS for the capacitated shelters case.

\begin{tabular}{ccc}
\hline & Istanbul European & Istanbul Anatolian \\
\hline WSS & $9,947,393$ & 35,952 \\
EVPI & $1,658,300$ & 13,546 \\
VSS & $\infty$ & $\infty$ \\
\hline
\end{tabular}

Percentage Evacuated, Scenario 11, Istanbul European

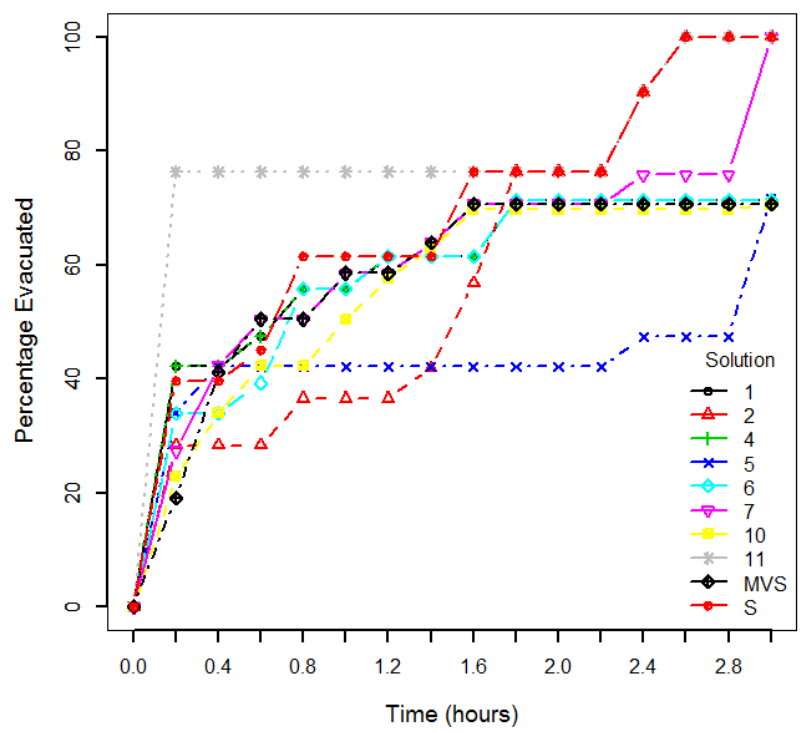

Percentage Evacuated, Scenario 7, Istanbul Anatolian

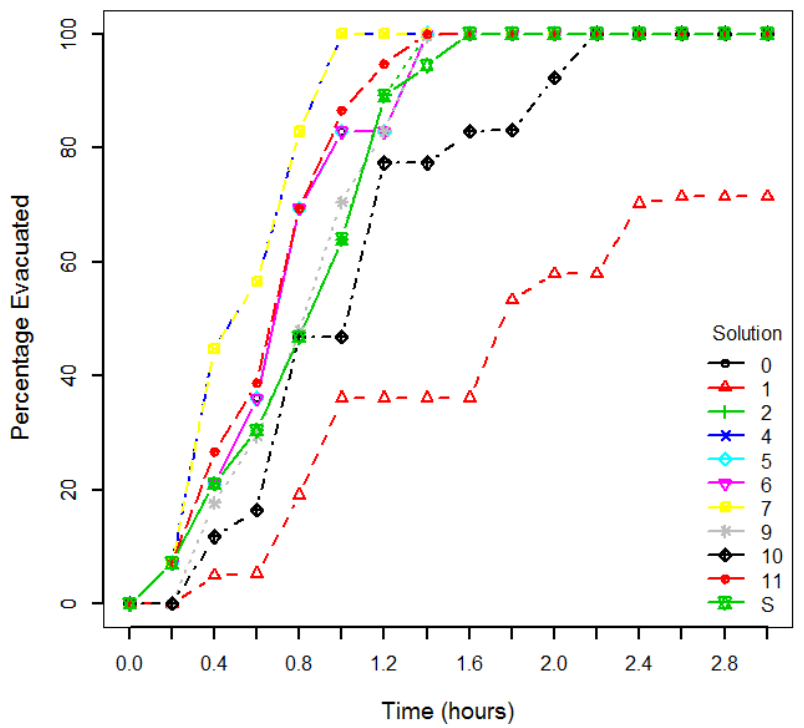

FIGURE 3 . The percentage of evacuees reaching safety across time for different strategies when shelters are capacitated.

For both Istanbul European and Anatolian networks the value of stochastic solution is infinite. We also observe that planning according to a single scenario leads to much worse performance compared to the case when shelters are uncapacitated. Whichever scenario is chosen for evacuation planning purposes, the solution becomes infeasible for some scenarios in both Istanbul European and Anatolian networks. For that reason the maximum regret is infinite for all other solutions except that of the stochastic programming solution for both Istanbul European and Anatolian networks.

Figure 3 depicts the percentage of evacuees reaching safety across time for different strategies, for scenario 7 of Istanbul Anatolian instance, when $p=17$ and $\lambda=0.2$ and for scenario 11 of Istanbul European instance when $p=32$ and $\lambda=0.1$. For the Istanbul European instance, stochastic programming solution is the second best strategy after strategy 11 in scenario 11. Everyone is evacuated to safety in less than 3 hours with stochastic programming solution. Only a little bit more than $20 \%$ of evacuees incur a latency of more than 2.8 hours. The mean value solution is not promising compared to the efficiency of the stochastic programming solution. The situation is similar for the Istanbul Anatolian instance. Stochastic programming solution turns out to be an efficient solution in this scenario, as well. With stochastic programming solution almost $90 \%$ of evacuees reach safety in less than 1.2 hours and only less than $5 \%$ of the population incurs a latency of more than 1.4 hours. Everyone is evacuated to safety in less than 1.6 hours. As the mean value solution is not feasible in this scenario, it is not in the graph. Table 15 illustrates the maximum latency incurred by the evacuees for different solution strategies across scenarios for Istanbul Anatolian network, when $p=17$ and $\lambda=0.2$. The stochastic programming solution has relatively a good maximum latency in most of the scenarios. For some scenarios the 


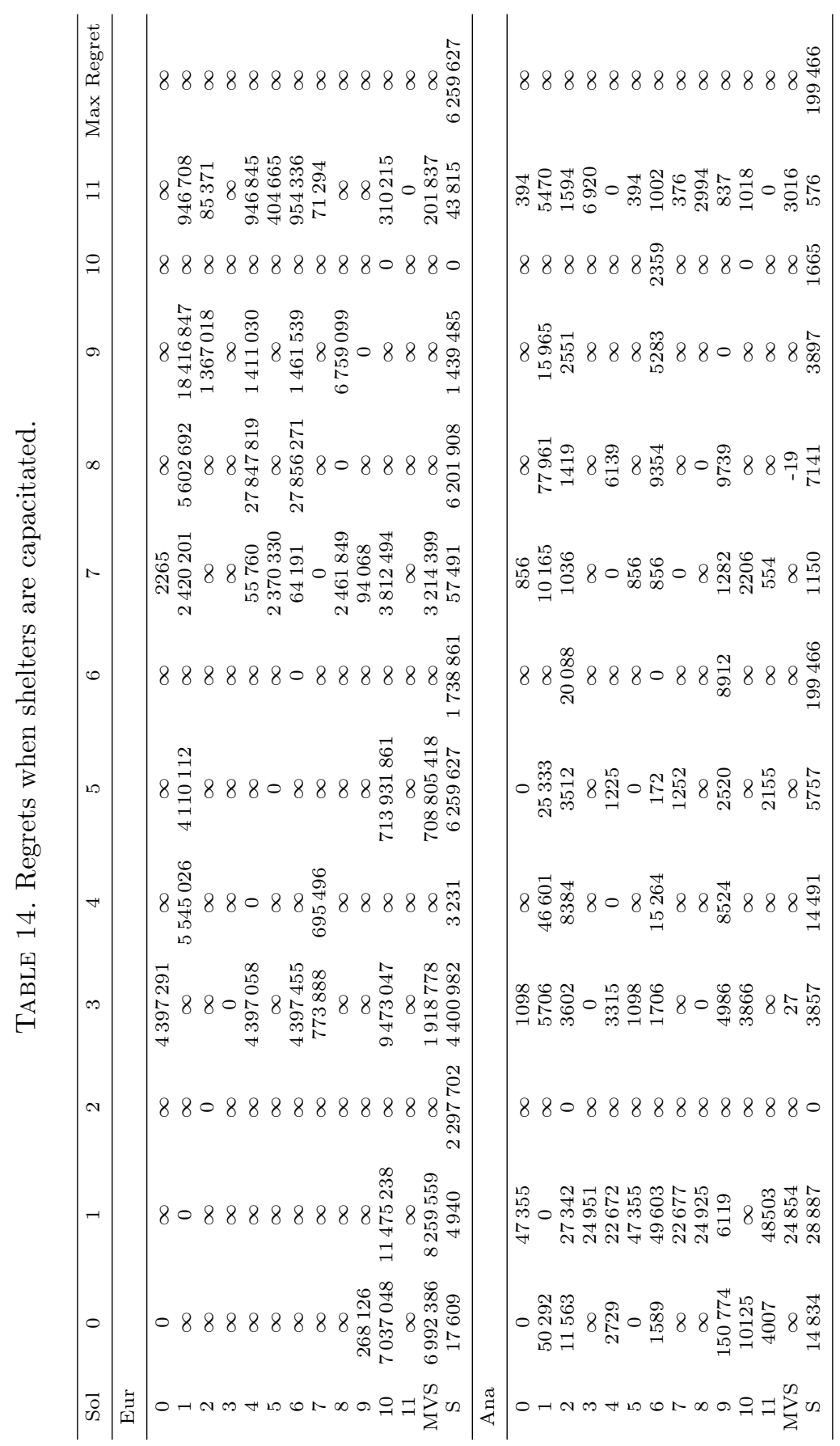


TABLE 15. Maximum Latency when shelters are capacitated, Istanbul Anatolian, $\lambda=0.2$ and $p=17$.

\begin{tabular}{|c|c|c|c|c|c|c|c|c|c|c|c|c|c|}
\hline & Scen & 0 & 1 & 2 & 3 & 4 & 5 & 6 & 7 & 8 & 9 & 10 & 11 \\
\hline \multicolumn{14}{|c|}{ Solution } \\
\hline 0 & & 0.20 & 4.10 & $\infty$ & 0.21 & $\infty$ & 0.25 & $\infty$ & 0.14 & $\infty$ & $\infty$ & $\infty$ & 0.16 \\
\hline 1 & & 1.51 & 0.97 & $\infty$ & 0.34 & 1.40 & 1.08 & $\infty$ & 0.78 & 1.74 & 0.70 & $\infty$ & 0.46 \\
\hline 2 & & 0.68 & 4.10 & 2.22 & 0.63 & 0.73 & 0.36 & 2.04 & 0.15 & 0.56 & 0.42 & $\infty$ & 0.19 \\
\hline 3 & & $\infty$ & 4.10 & $\infty$ & 0.15 & $\infty$ & $\infty$ & $\infty$ & $\infty$ & $\infty$ & $\infty$ & $\infty$ & 0.71 \\
\hline 4 & & 0.51 & 4.10 & $\infty$ & 0.63 & 0.45 & 0.36 & $\infty$ & 0.09 & 0.64 & $\infty$ & $\infty$ & 0.11 \\
\hline 5 & & 0.20 & 4.10 & $\infty$ & 0.21 & $\infty$ & 0.25 & $\infty$ & 0.14 & $\infty$ & $\infty$ & $\infty$ & 0.16 \\
\hline 6 & & 0.24 & 4.10 & $\infty$ & 0.25 & 1.04 & 0.25 & 0.64 & 0.14 & 0.64 & 0.59 & 22.18 & 0.17 \\
\hline 7 & & $\infty$ & 4.10 & $\infty$ & $\infty$ & $\infty$ & 0.36 & $\infty$ & 0.09 & $\infty$ & $\infty$ & $\infty$ & 0.16 \\
\hline 8 & & $\infty$ & 4.10 & $\infty$ & 0.15 & $\infty$ & $\infty$ & $\infty$ & $\infty$ & 0.63 & $\infty$ & $\infty$ & 0.36 \\
\hline 9 & & 6.07 & 1.50 & $\infty$ & 0.63 & 0.73 & 0.36 & 1.85 & 0.15 & 0.64 & 0.42 & $\infty$ & 0.15 \\
\hline 10 & & 0.53 & $\infty$ & $\infty$ & 0.63 & $\infty$ & $\infty$ & $\infty$ & 0.20 & $\infty$ & $\infty$ & 22.18 & 0.14 \\
\hline 11 & & 0.51 & 4.10 & $\infty$ & $\infty$ & $\infty$ & 0.36 & $\infty$ & 0.12 & $\infty$ & $\infty$ & $\infty$ & 0.11 \\
\hline MVS & & $\infty$ & 4.09 & $\infty$ & 0.15 & $\infty$ & $\infty$ & $\infty$ & $\infty$ & 0.62 & $\infty$ & $\infty$ & 0.36 \\
\hline $\mathrm{S}$ & & 0.68 & 4.09 & 2.22 & 0.63 & 0.73 & 0.36 & 8.87 & 0.14 & 0.64 & 0.42 & 22.16 & 0.14 \\
\hline
\end{tabular}

TABLE 16. Shelter Utilization when shelters are capacitated, Istanbul Anatolian.

\begin{tabular}{|c|c|c|c|c|c|c|c|c|c|c|c|c|c|c|c|c|c|}
\hline \multicolumn{18}{|c|}{ Shelters } \\
\hline Scenario & 14 & 15 & 16 & 17 & 18 & 19 & 20 & 21 & 22 & 23 & 24 & 25 & 26 & 27 & 28 & 29 & 30 \\
\hline 0 & 1785 & 0 & 0 & 4712 & 1636 & 30,000 & 0 & $\mathrm{D}$ & 0 & 12,938 & 3013 & 0 & 15,820 & 0 & 278 & 0 & 4784 \\
\hline 1 & 1496 & 0 & 0 & 4692 & 1491 & 19,990 & 0 & 6477 & 0 & 10,380 & 177 & 0 & 14,030 & 0 & 276 & 0 & 4254 \\
\hline 2 & 2386 & 0 & $\mathrm{D}$ & 9673 & 6913 & 27,657 & 0 & 8769 & 0 & 17,925 & 21,639 & 0 & $\mathrm{D}$ & 0 & 352 & 0 & 6170 \\
\hline 3 & 825 & 0 & 0 & $\mathrm{D}$ & 6687 & 9949 & 0 & 10,000 & 0 & $\mathrm{D}$ & 1447 & 0 & 9816 & 0 & 168 & 0 & 3031 \\
\hline 4 & 1413 & 395 & 0 & 6462 & 4390 & 14,798 & 0 & 10,000 & 0 & 13,199 & 3084 & 0 & 16,709 & 0 & 277 & 0 & 4860 \\
\hline 5 & 1732 & 0 & 0 & 7816 & 6549 & 17,633 & 0 & 1654 & 0 & 11,204 & 1224 & 0 & 13,743 & 0 & 253 & 0 & 4392 \\
\hline 6 & 2512 & 0 & 0 & 11,514 & 9330 & 25,978 & 0 & 6473 & 0 & 17,221 & 1427 & 0 & 22,230 & 0 & 385 & 0 & 6638 \\
\hline 7 & 905 & 0 & $\mathrm{D}$ & 4918 & $\mathrm{D}$ & 14,528 & 0 & $\mathrm{D}$ & $\mathrm{D}$ & 7126 & 1790 & 0 & 9399 & 0 & 159 & 0 & 2775 \\
\hline 8 & 1755 & 0 & 0 & 4918 & 0 & 14,528 & 0 & 0 & 0 & 7126 & 1790 & 0 & 9399 & 0 & 159 & 0 & 2775 \\
\hline 9 & 1533 & 0 & 0 & 10,920 & 8820 & $\mathrm{D}$ & 0 & 10,000 & 0 & 11,711 & 1028 & 0 & 14,029 & 0 & 234 & 3860 & 4317 \\
\hline 10 & $\mathrm{D}$ & 463 & 2049 & $\mathrm{D}$ & 8286 & 28,867 & $\mathrm{D}$ & 10,001 & 0 & 0 & 15,684 & $\mathrm{D}$ & 25,000 & 0 & 342 & 0 & 6,421 \\
\hline 11 & 951 & 0 & 0 & 4,698 & 1123 & 7891 & 0 & 7557 & 0 & 6624 & 2 & 0 & 8164 & 0 & 174 & 0 & 2376 \\
\hline Max & 2512 & 463 & 2049 & 11,514 & 9,330 & 30,000 & 0 & 10,001 & 0 & 17,925 & 21,639 & 0 & 25,000 & 0 & 385 & 3860 & 6638 \\
\hline Min & 825 & 0 & 0 & 4692 & 0 & 7891 & 0 & 0 & 0 & 0 & 2 & 0 & 8164 & 0 & 159 & 0 & 2376 \\
\hline Ave & 1572 & 71 & 205 & 7032 & 5021 & 19,256 & 0 & 7093 & 0 & 10,496 & 4359 & 0 & 14,394 & 0 & 255 & 322 & 4399 \\
\hline
\end{tabular}

maximum latency incurred in stochastic programming solution is the same as that of the solution strategy that considers the related scenario. Stochastic programming solution provides better results than those of the mean value solution, as the mean value solution is not feasible for many scenarios. The infeasibility of other solution strategies in many scenarios, shows the superiority of the stochastic programming solution.

In Table 16, we report the utilization numbers of the shelters for Istanbul Anatolian instance with $p=17$ and $\lambda=0.2$ for stochastic programming solution across different scenarios, when shelters are capacitated. We also report the maximum, minimum and the average utilization numbers. Among the 17 potential shelters, only 13 are selected to be opened in the optimal solution. The four shelters that are not opened in the optimal solution, are shelters 20,22, 25, and 27. Because of the restrictions on the shelter capacities, the evacuees are distributed more evenly between shelters compared to the uncapacitated case, at the cost of an increase in total evacuation time.

\section{Conclusion}

Evacuation planning is done in the presence of uncertainty without exact or complete information. Due to the fact that the place, the time and the scale of a disaster can not be easily predicted, it is very difficult to estimate 
the evacuation demand accurately. Similarly, road capacities and shelter sites may be affected (degraded or disrupted), but the questions of "which ones" and "how much" are difficult to answer.

In this study, we first proposed a novel model that captures this stochasticity in evacuation planning by taking account of the uncertainty in demand, road capacities and availability of shelters. Following the methodology proposed by Bayram et al. [8], we showed that our problem can be solved exactly by using second order cone programming in small CPU times.

Then we presented a real world case study for a pending Istanbul earthquake where evacuation decisions are made to save people from the impact of aftershocks and/or secondary disasters. In our case study, we observed that planning in accordance with a single scenario such as the worst case scenario or most probable scenario, or solving the problem with mean value approach do not produce effective solutions and that using stochastic programming can lead to significant improvements. The improvement ranges from 2 to 33 and 4 to 15 fold in total evacuation time and maximum latency respectively, depending on the tolerance level used. We observed that stochastic programming solution results in significantly better maximum regret values ranging from 7 to 70 times compared to deterministic solutions, when shelters are uncapacitated. We showed that if the fairness considerations are applied using geographical distances rather than travel times, contrary to common managerial belief it is not necessarily more beneficial to open more shelters as this may cause congestion on the road network. We also showed as a managerial rule of thumb that for the case when shelters are uncapacitated, evacuation planning authority should take into account maximum utilization numbers of shelters to determine shelter capacities. The solutions provided by stochastic programming approach is robust across the range of scenarios in terms of the high percentages of the population evacuated within a certain time limit. Depending on the realized scenario, the tolerance level used and the decision on the number of shelters to open, stochastic programming solution is able evacuate around $90 \%$ of the evacuees within 5 hours and everyone is evacuated to safety within 6 hours for the Istanbul Anatolian instances. The results for Istanbul European instances are as significant as those of Istanbul Anatolian instances.

We further observed that when shelters are capacitated, the superiority of stochastic programming solution is emphasized, as planning in accordance with a single scenario or mean value approach will possibly generate infeasible solutions for some scenarios, unlike in stochastic programming solution.

In this study, we were able to solve the stochastic programs in small CPU times as the number of scenarios was small. In a follow up study (Bayram and Yaman [9]), we work on decomposition approaches to solve the problem with large number of scenarios.

Specifying which routes are going play a major role during an evacuation is a question emergency evacuation planners are trying to answer. It is important that these routes are large enough to serve a demand surge during an evacuation and that they survive the destructive impact of a disaster. For that reason expanding and/or strengthening these routes subject to a budget would contribute to the effectiveness of an evacuation plan. As a continuation of our study we aim to incorporate capacity expansion and/or retrofit decisions in the first stage and analyse the trade off between the expansion/retrofit decisions and the total evacuation time.

In our model the uncertainty is caused by nature. Another interesting extension would be a game theoretic approach, where there is an attacker (a terrorist organization using a chemical/biological bomb) whose aim is to give the defender maximum possible harm by delaying the evacuation as much as possible by attacking critical road segments or bridges and shelter sites subject to a limited budget. Defender on the other hand, tries to minimize this effect by selecting the shelter locations and assigning the evacuees to these shelters and to routes that reach them in such a way that the total evacuation time is minimized.

Acknowledgements. This research is supported by TUBITAK grant no. 213M434. The research of the second author is supported by the Turkish Academy of Sciences. 


\section{REFERENCES}

[1] L. Alçada-Almeida, L. Tralhão, L. Santos and J. Coutinho-Rodrigues, A multiobjective approach to locate emergency shelters and identify evacuation routes in urban areas. Geographical Anal. 41 (2009) 9-29.

[2] ARC. Standards for hurricane evacuation shelter selection, arc 4496. Technical report, American Red Cross (2002).

[3] S.A. Ardekani and A.G. Hobeika, Logistics problems in the aftermath of the 1985 mexico city earthquake. In vol. 42 of Transportation Quarterly (1988).

[4] E.J. Baker, Hurricane evacuation behavior. Inter. J. Mass Emergencies Disasters. 9 (1991) 287-310.

[5] B. Balçık and B.M. Beamon, Facility location in humanitarian relief. Inter. J. Logistics 11 (2008) 101-121.

[6] G. Barbarosoğlu and Y. Arda, A two-stage stochastic programming framework for transportation planning in disaster response. J. Oper. Res. Soc. 55 (2004) 43-53.

[7] V. Bayram, Optimization models for large scale network evacuation planning and management: A literature review. Surv. Oper. Res. Manag. Sci. 21 (2016) 63-84.

[8] V. Bayram, B.Ç. Tansel and H. Yaman, Compromising system and user interests in shelter location and evacuation planning. Trans. Res. B: Methodol. 72 (2015) 146-163.

[9] V. Bayram and H. Yaman, Shelter location and evacuation route assignment under uncertainty: A benders decomposition approach. Trans. Sci. 52 (2017) 416-436.

[10] S. Belardo, J. Harrald, W.A. Wallace and J. Ward, A partial covering approach to siting response resources for major maritime oil spills. Manag. Sci. 30 (1984) 1184-1196.

[11] N. Berktaş, B.Y. Kara and O.E. Karaşan, Solution methodologies for debris removal in disaster response. EURO J. Comput. Optimiz. 4 (2016) 403-445.

[12] O. Berman, R. Huang, S. Kim and M.B. Menezes, Locating capacitated facilities to maximize captured demand. IIE Trans. 39 (2007a) 1015-1029.

[13] O. Berman and D. Krass, Facility location problems with stochastic demands and congestion. Facility Location Applications and Theory (2002).

[14] O. Berman, D. Krass and M.B. Menezes, Facility reliability issues in network p-median problems: Strategic centralization and co-location effects. Oper. Res. 55 (2007b) 332-350.

[15] O. Berman, D. Krass and M.B. Menezes, Locating facilities in the presence of disruptions and incomplete information. Decision Sci. 40 (2009) 845-868.

[16] O. Berman and A.R. Odoni, Locating mobile servers on a network with markovian properties. Networks 12 (1982) 73-86.

[17] Berman, O. and Wang, J., The 1-median and 1-antimedian problems with uniform distributed demands. Technical report, Working paper, Rotman School of Management, University of Toronto, Toronto, Canada (2004).

[18] J.R. Birge and F. Louveaux, Introduction to stochastic programming. Springer (1997).

[19] D. Bish, H. Sherali and A. Hobeika, Optimal evacuation planning using staging and routing. J. Oper. Res. Soc. 65 (2013) 124-140.

[20] S. Bretschneider, Mathematical models for evacuation planning in urban areas, vol. 659. Springer (2013).

[21] S. Bretschneider and A. Kimms, A basic mathematical model for evacuation problems in urban areas. Trans. Res. part A: Policy and Practice 45 (2011) 523-539.

[22] M. Bundschuh, D. Klabjan and D.L. Thurston, Modeling robust and reliable supply chains. Optimization Online Available at: http://www.optimization-online.org/DB_HTML/2003/07/679.html (2003).

[23] T.H. Byers and M.S. Waterman, Technical note, Determining all optimal and near-optimal solutions when solving shortest path problems by dynamic programming. Oper. Res. 32 (1984)1381-1384.

[24] A.M. Caunhye, X. Nie and S. Pokharel, Optimization models in emergency logistics: A literature review. Socio-Econ. Plan. Sci. 46 (2012) 4-13.

[25] M. Çelik, Ö. Ergun and P. Keskinocak, The post-disaster debris clearance problem under incomplete information. Oper. Res. 63 (2015) 65-85.

[26] Y. Chan, W.B. Carter and M.D. Burnes, A multiple-depot, multiple-vehicle, location-routing problem with stochastically processed demands. Comput. Oper. Res. 28 (2001) 803-826.

[27] M.-S. Chang, Y.-L. Tseng and J.-W. Chen, A scenario planning approach for the flood emergency logistics preparation problem under uncertainty. Transp. Res. Part E: Logistics Trans. Rev. 43 (2007) 737-754.

[28] N.-B. Chang and S. Wang, A locational model for the site selection of solid waste management facilities with traffic congestion constraints. Civil Engineering Syst. 11 (1995) 287-306.

[29] G. Chen, M.S. Daskin, Z.-J.M. Shen and S. Uryasev, The $\alpha$-reliable mean-excess regret model for stochastic facility location modeling. Nav. Res. Logist. (NRL) $\mathbf{5 3}$ (2006) 617-626.

[30] Y.C.Chiu, H. Zheng, J. Villalobos and B. Gautam, Modeling no-notice mass evacuation using a dynamic traffic flow optimization model. IIE Trans. 39 (2007) 83-94.

[31] CNN. Cnn live at daybreak: Escaping a hurricane. http://edition.cnn.com/TRANSCRIPTS/ (2001).

[32] L. Cooper, A random locational equilibrium problem. J. Regional Sci. 14 (1974) 47-54.

[33] J. Coutinho-Rodrigues, L. Tralhão and L. Alçada-Almeida, Solving a location-routing problem with a multiobjective approach: the design of urban evacuation plans. J. Transp. Geography 22 (2012) 206-218.

[34] T.J. Cova and J.P. Johnson, A network flow model for lane-based evacuation routing. Transp. Res. Part A: Policy and Practice 37 (2003) 579-604. 
[35] T. Cui, Y. Ouyang and Z.-J.M. Shen, Reliable facility location design under the risk of disruptions. Oper. Res. 58 (2010) 998-1011.

[36] N. Dash and H. Gladwin, Evacuation decision making and behavioral responses: Individual and household. Natural Hazards Rev. 8 (2007) 69-77.

[37] M.S.Daskin, C.R. Coullard and Z.-J.M. Shen, An inventory-location model: Formulation, solution algorithm and computational results. Ann. Oper. Res. 110 (2002).83-106.

[38] S. Duran, M.A. Gutierrez and P. Keskinocak, Pre-positioning of emergency items for care international. Interfaces 41 (2011) 223-237.

[39] H.A. Eiselt, M. Gendreau and G. Laporte, Location of facilities on a network subject to a single-edge failure. Networks 22 (1992) 231-246.

[40] FEMA. Handbook for the seismic evaluation of buildings, fema 310. Technical report, Federal Emergency Management Agency (1988).

[41] FEMA. Risk management series, safe rooms and shelters: Protecting people against terrorist attacks, fema 453. Technical report, Federal Emergency Management Agency (2006).

[42] FEMA. Public assistance debris management guide, https://www.fema.gov/pdf/government/grant/pa/demagde.pdf . Technical report, Federal Emergency Management Agency (2007).

[43] FEMA. Design and construction guidance for community safe rooms, fema p-361, second edition. Technical report, Federal Emergency Management Agency (2008).

[44] H. Frank, Optimum locations on a graph with probabilistic demands. Oper. Res. 14 (1966) 409-421.

[45] G. Galindo and R. Batta, Review of recent developments in OR/MS research in disaster operations management. Eur. J. Oper. Res. (2013).

[46] N. Görmez, M. Köksalan and F. Salman, Locating disaster response facilities in istanbul. J. Oper. Res. Soc. 62 (2011) $1239-1252$.

[47] D. Günneç and F.S. Salman, Assessing the reliability and the expected performance of a network under disaster risk. OR Spectrum 33 (2011) 499-523.

[48] H.W. Hamacher and S.A. Tjandra, Mathematical modelling of evacuation problems-a state of the art. Pedestr. Evac. Dyn. 2002 (2002) 227-266.

[49] R. Hasegawa, Disaster evacuation from japans 2011 tsunami disaster and the fukushima nuclear accident. IDDRI Study No5 (2013).

[50] A.G. Hobeika, Transportation evacuation planning under natural disasters. In 10th International Road Federation World Meeting, Rio de Janerio (1984).

[51] R. Huang, S. Kim and M.B. Menezes, Facility location for large-scale emergencies. Ann. Oper. Res. 181 (2010) $271-286$.

[52] O. Huibregtse, S. Hoogendoorn and M.C. Bliemer, Optimization of evacuation measures under uncertainty. In Transportation Research Board 89th Annual Meeting, number (2010) 10-2550.

[53] IMM-JICA. The study on a disaster prevention / mitigation basic plan in Istanbul including seismic microzonation in the Republic of Turkey. Technical report, The Istanbul Metropolitan Municipality Japan International Cooperation Agency (2002).

[54] A. Ingolfsson, S. Budge and E. Erkut, Optimal ambulance location with random delays and travel times. Health Care Manag. Sci. 11 (2008) 262-274.

[55] O. Jahn, R.H. Möhring, A.S. Schulz and N.E. Stier-Moses, System-optimal routing of traffic flows with user constraints in networks with congestion. Oper. Res. 53 (2005) 600-616.

[56] G. Kalafatas and S. Peeta, Planning for evacuation: insights from an efficient network design model. J. Infrastructure Syst. 15 (2009) 21-30.

[57] J. Kim and S.S. Oh, Confidence, knowledge, and compliance with emergency evacuation. J. Risk Res. 18 (2015) $111-126$.

[58] C. Kırıkçı, Determination of shelter locations and evacuation routes for a possible earthquake in the city of Istanbul. Master's thesis, Department of Industrial Engineering, Bilkent University (2012).

[59] S. Kongsomsaksakul, C. Yang and A. Chen, Shelter location-allocation model for flood evacuation planning. J. East. Asia Soc. Transp. Stud. 6 (2005) 4237-4252.

[60] A. Kulshrestha, D. Wu, Y. Lou and Y. Yin, Robust shelter locations for evacuation planning with demand uncertainty. J. Transp. Safety Secur. 3 (2011) 272-288.

[61] G. Laporte, F. Louveaux and H. Mercure, Models and exact solutions for a class of stochastic location-routing problems. Eur. J. Oper. Res. 39 (1989) 71-78.

[62] A.C.Y. Li, L. Nozick, N. Xu and R. Davidson, Shelter location and transportation planning under hurricane conditions. Transp. Res. Part E: Logist. Transp. Rev. 48 (2012) 715-729.

[63] X. Li and Y. Ouyang, A continuum approximation approach to reliable facility location design under correlated probabilistic disruptions. Transp. Res. Part B: Methodological 44 (2010) 535-548.

[64] M. Lim, M.S. Daskin, A. Bassamboo and S. Chopra, A facility reliability problem: formulation, properties, and algorithm. Nav. Res. Logist. (NRL) $\mathbf{5 7}$ (2010) 58-70.

[65] M.K. Lindell and C.S. Prater, Critical behavioral assumptions in evacuation time estimate analysis for private vehicles: Examples from hurricane research and planning. J. Urban Plan. Dev. 133 (2007) 18-29.

[66] F. Louveaux, Discrete stochastic location models. Ann. Oper. Res. 6 (1986) 21-34.

[67] L. Luther, Disaster debris removal after hurricane katrina: status and associated issues. Congressional Research Service, Library of Congress (2006). 
[68] A. Madansky, Inequalities for stochastic linear programming problems. Manag. Sci. 6 (1960) 197-204.

[69] K.D. Marano, D.J. Wald and T.I. Allen, Global earthquake casualties due to secondary effects: a quantitative analysis for improving rapid loss analyses. Nat. Hazards 52 (2010) 319-328.

[70] A.R. Mawson, Understanding mass panic and other collective responses to threat and disaster. Psychiatry: Interpers. Biol. Process. 68 (2005) 95-113.

[71] H.O. Mete and Z.B. Zabinsky, Stochastic optimization of medical supply location and distribution in disaster management. Inter. J. Prod. Econ. 126 (2010) 76-84.

[72] P.B. Mirchandani and A.R. Odoni, Locations of medians on stochastic networks. Trans. Sci. 13 (1979) 85-97.

[73] P.B. Mirchandani and A. Oudjit, Localizing 2-medians on probabilistic and deterministic tree networks. Networks 10 (1980) 329-350.

[74] P. Murray-Tuite and B. Wolshon, Evacuation transportation modeling: An overview of research, development, and practice. Transp. Res. Part C: Emerging Technologies 27 (2013) 25-45.

[75] L.D. Nel and C.J. Colbourn, Locating a broadcast facility in an unreliable network. INFOR 28 (1990) $363-379$.

[76] A. Nemirovski and A.B. Tal, Lectures on modern convex optimization: Analysis, algorithms, and engineering applications. MPS-SIAM Series on Optimization (2001).

[77] M. Ng and S.T. Waller, Reliable evacuation planning via demand inflation and supply deflation. Transp. Res. Part E: Logistics and Transp. Rev. 46 (2010) 1086-1094.

[78] N. Noyan, Risk-averse two-stage stochastic programming with an application to disaster management. Comput. Oper. Res. 39 (2012) 541-559.

[79] A.J. Pel, M.C.J. Bliemer and S.P. Hoogendoorn, A review on travel behaviour modelling in dynamic traffic simulation models for evacuations. Transp. 39 (2012) 97-123.

[80] P. Peng, L.V. Snyder, A. Lim and Z. Liu, Reliable logistics networks design with facility disruptions. Transp. Res. Part B: Methodological 45 (2011) 1190-1211.

[81] R.W. Perry, Evacuation decision-making in natural disasters. Mass Emergencies 4 (1979) 25-38.

[82] R.W. Perry and M.K. Lindell, Preparedness for emergency response: guidelines for the emergency planning process. Disasters 27 (2003) 336-350.

[83] H.N. Psaraftis, G.G. Tharakan and A. Ceder, Optimal response to oil spills: the strategic decision case. Oper. Res. 34 (1986) 203-217.

[84] C.G. Rawls and M.A. Turnquist, Pre-positioning of emergency supplies for disaster response. Trans. Res. Part B: Methodological 44 (2010) 521-534.

[85] S. Rui, H. Shiwei and L. Zhang, Optimum transit operations during the emergency evacuations. J. Trans. Syst. Eng. Information Techn. 9 (2009) 154-160.

[86] S. Sen, Algorithms for stochastic mixed-integer programming models. Handbooks in Oper. Res. Manag. Sci. 12 (2005) 515-558.

[87] Y. Sheffi, Urban transportation networks: equilibrium analysis with mathematical programming methods. Prentice-Hall, Inc., Englewood Cliffs, N.J. 07632 (1985).

[88] Z.-J.M. Shen, J. Pannala, R. Rai and T.S. Tsoi, Modeling transportation networks during disruptions and emergency evacuations. University of California Transportation Center (2008).

[89] H.D. Sherali, T.B. Carter and A.G. Hobeika, A location-allocation model and algorithm for evacuation planning under hurricane/flood conditions. Trans. Res. Part B: Methodological 25 (1991) 439-452.

[90] J.-B. Sheu and C. Pan, A method for designing centralized emergency supply network to respond to large-scale natural disasters. Trans. Res. Part B: Methodological 67 (2014) 284-305.

[91] L.V. Snyder and M.S. Daskin, Reliability models for facility location: the expected failure cost case. Trans. Sci. 39 (2005) 400-416.

[92] L.V. Snyder and M.S. Daskin, Models for reliable supply chain network design. In Critical Infrastructure. Springer (2007) $257-289$.

[93] L.V. Snyder, M.P. Scaparra, M.S.Daskin and R.L. Church, Planning for disruptions in supply chain networks. Tutorials Oper. Res. (2006) 234-257.

[94] A. Stepanov and J.M. Smith, Multi-objective evacuation routing in transportation networks. Eur. J. Oper. Res. 198 (2009) 435-446.

[95] TAM. Traffic Assignment Manual. Bureau of Public Roads, U.S. Department of Commerce (1964).

[96] TRB, Transportation Research Board of the National Academies, C. o. t. R. o. P. T. i. E.E. The role of transit in emergency evacuation. Special Report (2008) 294. http://www.TRB.org/.

[97] H. Tüydeş, Network Traffic Management under Disaster Conditions. Ph.D. thesis, Field of Civil and Environmental Engineering, Northwestern University (2005).

[98] W. Wilhelm and A.V. Srinivasa, A strategic, area-wide contingency planning model for oil spill cleanup operations with application demonstrated to the galveston bay area. Decision Sci. 27 (1996) 767-799.

[99] T. Yamada, A network flow approach to a city emergency evacuation planning. Inter. J. Syst. Sci. 27 (1996) 931-936.

[100] T. Yao, S.R. Mandala and B. Do Chung, Evacuation transportation planning under uncertainty: a robust optimization approach. Networks Spatial Econ. 9 (2009) 171-189.

[101] M.A. Yazıcı and K. Özbay, Evacuation network modeling via dynamic traffic assignment with probabilistic demand and capacity constraints. Trans. Res. Record: J. Transp. Res. Board 2196 (2010) 11-20. 